\title{
Exact Non-Reflecting Boundary Conditions on Perturbed Domains and $h p$-Finite Elements
}

\author{
Tommy L. Binford, Jr. • David P. Nicholls • \\ Nilima Nigam · T. Warburton
}

Received: 15 February 2008 / Revised: 16 December 2008 / Accepted: 16 December 2008 /

Published online: 6 January 2009

(C) Springer Science+Business Media, LLC 2008

\begin{abstract}
For exterior scattering problems one of the chief difficulties arises from the unbounded nature of the problem domain. Inhomogeneous obstacles may require a volumetric discretization, such as the Finite Element Method (FEM), and for this approach to be feasible the exterior domain must be truncated and an appropriate condition enforced at the far, artificial, boundary. An exact, non-reflecting boundary condition can be stated using the classical DtN-FE method if the Artificial Boundary's shape is quite specific: circular or elliptical. Recently, this approach has been generalized to permit quite general Artificial Boundaries which are shaped as perturbations of a circle resulting in the "Enhanced DtN-FE" method. In this paper we extend this method to a two-dimensional FEM featuring high-order polynomials in order to realize a high rate of convergence. This is more involved than simply specifying high-order test and trial functions as now the scatterer shape and Artificial Boundary must be faithfully represented. This entails boundary elements which conform (to high order) to the true boundary shapes. As we show, this can be accomplished and we realize an arbitrary order FEM without spurious reflections.
\end{abstract}

Keywords Non-reflecting boundary conditions · $h p$-finite elements · Acoustic scattering • Dirichlet-to-Neumann maps · Geometric perturbation methods

\section{Introduction}

The scattering of time-harmonic linear acoustic waves from a bounded obstacle arises in a wide array of applications of great importance to scientists and engineers. Such applications

T.L. Binford, Jr. · T. Warburton

Computational and Applied Mathematics, Rice University, Houston, TX 77005, USA

D.P. Nicholls $(\bowtie)$

Department of Mathematics, Statistics, and Computer Science, University of Illinois at Chicago,

Chicago, IL 60607, USA

e-mail: nicholls@math.uic.edu

N. Nigam

Department of Mathematics, Simon Fraser University, Burnaby, BC V5A 1S6, Canada 
include remote sensing and non-destructive testing. Despite the linear nature of the governing partial differential equations, several inherent features of these applications give rise to many difficult algorithmic and theoretical challenges. Among these is the fundamentally unbounded nature of the problem domain which presents a particular difficulty for numerical simulations.

In many situations an integral equation method can be readily applied in the frequency domain [8] which not only posits near-field unknowns, but also satisfies the far-field condition, the so-called Sommerfeld Radiation Condition, exactly via an astute choice of integral kernel. However, some applications, such as an inhomogeneous obstacle with a discontinuous index of refraction, require a volumetric discretization; here Finite Element Methods (FEM) are particularly appealing due to their geometric flexibility, reliability, and the widespread availability of FEM software. In a volumetric discretization an Artificial Boundary is typically introduced to the computational domain (exterior to the scatterer) to render it bounded, and an approximation of the Sommerfeld Radiation Condition [8] is enforced there. There is a large literature of such methods and we refer the interested reader to the excellent survey book of Ihlenburg [19].

Of particular relevance to the present paper is the work in the frequency domain of Feng [10], Han and Wu [16], and Keller and Givoli [11-14, 20] (please see [19] for a complete literature survey). In these the shape of the Artificial Boundary must be chosen to be quite simple: circular or elliptical (spherical or ellipsoidal in three dimensions), i.e. a separable geometry. In this case the solution exterior to the Artificial Boundary can be written exactly in terms of appropriate basis functions (e.g. Hankel functions), which can then be used to readily compute the Dirichlet-to-Neumann map (DtN map) enabling a "NonReflecting" (or "Transparent") boundary condition to be posed at the Artificial Boundary. An FEM linked to the Non-Reflecting boundary condition, featuring the DtN map, results in the DtN-FE method. This method, however, suffers from two important flaws: The DtN map is inherently non-local, and the Artificial Boundary has very specific shape requirements. The first results in the appearance of dense sub-blocks in the otherwise sparse linear system of equations that must be solved. The second results in a needlessly large computational domain if the scatterer cannot be efficiently enclosed by a circle or ellipse.

In a sequence of recent papers, two of the authors have addressed the latter of these concerns with a generalization of the DtN-FE method [28, 29]. This "Enhanced DtN-FE" method permits Artificial Boundaries which are shaped as perturbations of a circle and is based upon the fact that the DtN map depends analytically upon shape perturbation. With the wide range of shapes that one can specify in this way, this generalization allows one to fit the Artificial Boundary quite close to the surface of the scatterer. In [28] it was demonstrated that this method can be implemented and coupled to a piecewise linear FEM while not destroying its inherent convergence rate. In [29] it was shown that the resulting discrete problem is well-posed provided that the DtN map is suitably modified (see $[9,15,17]$ ). Moreover, this proof did not rely upon the piecewise linear nature of the underlying basis/test functions and thus can be applied equally well to other FEM. In this paper we extend this method to a two-dimensional FEM featuring high-order polynomials in order to realize a higher rate of convergence. This is more involved than simply specifying test and trial functions of higher polynomial order as now the scatterer shapes and Artificial Boundary must be faithfully represented, i.e. the boundary elements must conform (to high order) to the true boundary shapes. However, as we shall see in the numerical results of Sect. 4, this can be accomplished and we realize an arbitrary order FEM without spurious reflections. Additionally, we present in Appendix new formulas for the rapid execution of one popular algorithm for the approximation of DtN maps (the method of Operator Expansions) which plays a crucial role in our method. 
The organization of the paper is as follows: In Sect. 2 we review the governing equations of time-harmonic acoustic scattering by a bounded obstacle in the frequency domain, and the $h p$-FEM we employ. In Sect. 3 we recall the considerations necessary to compute the DtN map for use in our FEM scheme, and in Sect. 4 we present a representative set of numerical examples which display the accuracy, flexibility, and robustness of our new highorder, Enhanced DtN-FE method. Concluding remarks are given in Sect. 5.

\section{Governing Equations}

It is well-known [8] that if time-harmonic plane-wave acoustic radiation of the form

$$
v_{i}=e^{i k \alpha \cdot x}, \quad|\alpha|=1,
$$

is incident upon a bounded, impenetrable obstacle $\Sigma \subset \mathbf{R}^{2}$ (with boundary $\Gamma$ ) then the (reduced) scattered field $v=v(r, \theta)$ satisfies the scalar Helmholtz equation

$$
\Delta v+k^{2} v=0 \quad \text { in } \Omega_{\infty}:=\operatorname{Ext}(\Sigma)
$$

Of course, to realize a unique solution one must specify, in addition to the periodicity in $\theta$, boundary conditions at the scatterer and at infinity. For the former, we choose a Dirichlet condition

$$
\left.v\right|_{\Gamma}=-\left.v_{i}\right|_{\Gamma}=: \xi(\theta),
$$

which defines the generic Dirichlet data $\xi$ (a Neumann condition can be treated in an analogous manner). For the latter, we have the Sommerfeld Radiation Condition [8] (in two dimensions):

$$
\lim _{r \rightarrow \infty} r^{1 / 2}\left(\partial_{r} v-i k v\right)=0 .
$$

Gathering (2), (3), and (4) we have the equations governing the scattering of time-harmonic, acoustic plane-waves from an irregular, impenetrable, two-dimensional obstacle:

$$
\begin{aligned}
\Delta v+k^{2} v & =0 \quad \text { in } \Omega_{\infty} \\
\left.v\right|_{\Gamma} & =\xi \\
\lim _{r \rightarrow \infty} r^{1 / 2}\left(\partial_{r} v-i k v\right) & =0 .
\end{aligned}
$$

\subsection{A Transparent Boundary Condition}

One of the severe difficulties associated with the numerical simulation of the system (5) is the unbounded nature of the computational domain $\Omega_{\infty}$. In FEM discretizations (as with other volumetric techniques) this problem is usually addressed with the introduction of an "Artificial Boundary," say $\mathcal{B}$, which properly encloses the scatterer $\Sigma$, and the imposition there of some boundary conditions motivated by the Sommerfeld Radiation Condition (4). 
Fig. 1 Depiction of a scatterer, $\Sigma$, with boundary $\Gamma$ and unit exterior normal $n$ enclosed within the Artificial Boundary $\mathcal{B}$ with normal $N_{\mathcal{B}}$. The region $\Omega(\Sigma, \mathcal{B})$ is the intersection of the exterior of $\Sigma$ with the interior of $\mathcal{B}$

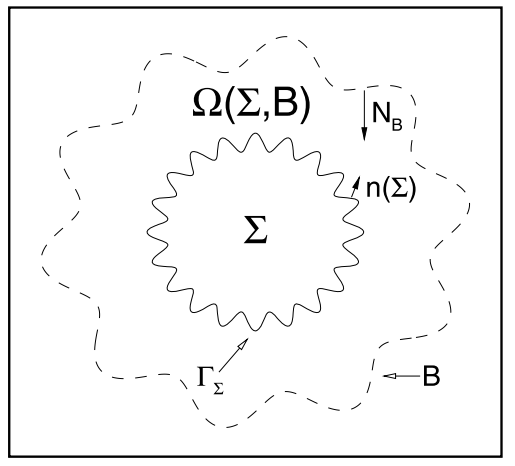

We now describe a "transparent" or "non-reflecting" boundary condition which can be enforced on such an Artificial Boundary. For this, consider the augmented scattering problem

$$
\begin{aligned}
\Delta v+k^{2} v & =0 & & \text { in } \Omega:=\Omega_{\infty} \backslash \operatorname{Ext}(\mathcal{B}) \\
v & =\xi & & \text { at } \Gamma \\
\partial_{N} v & =\partial_{N} w & & \text { at } \mathcal{B} \\
v & =w & & \text { at } \mathcal{B} \\
\Delta w+k^{2} w & =0 & & \text { in } \operatorname{Ext}(\mathcal{B}) \\
\lim _{r \rightarrow \infty} r^{1 / 2}\left(\partial_{r} w-i k w\right) & =0, & &
\end{aligned}
$$

where $N$ is a normal pointing into the interior of $\mathcal{B}$; please see Fig. 1. The solutions of (5) and (6) are identical in that the $v$ match on $\Omega$, and $v=w$ on the exterior of $\mathcal{B}$.

To specify the transparent boundary condition on $v$ at $\mathcal{B}$ consider (6d)-(6f):

$$
\begin{array}{rlrl}
\Delta w+k^{2} w & =0 \quad & & \text { in } \operatorname{Ext}(\mathcal{B}) \\
w & =\psi & \text { at } \mathcal{B} \\
\lim _{r \rightarrow \infty} r^{1 / 2}\left(\partial_{r} w-i k w\right) & =0, &
\end{array}
$$

where $\psi$ is meant to denote generic Dirichlet data at $\mathcal{B}$. Provided that $\mathcal{B}$ is sufficiently regular and $\psi$ sits in an appropriate function space (see, e.g., [8]), this system of equations has a unique solution. From this we can compute the quantity necessary to close (6a)-(6c), $\partial_{N} w$ at $\mathcal{B}$, i.e. the "Dirichlet-to-Neumann map" (DtN map)

$$
T(\mathcal{B})[\psi]:=\left.\nabla w\right|_{\mathcal{B}} \cdot N
$$

Therefore, based upon the augmented system (6), we can equivalently restate (5) on the bounded domain $\Omega$

$$
\begin{aligned}
\Delta v+k^{2} v=0 & & \text { in } \Omega \\
v=\xi & & \text { at } \Gamma \\
\partial_{N} v-T[v]=0 & & \text { at } \mathcal{B},
\end{aligned}
$$


with the transparent boundary condition at $\mathcal{B}$ specified via the non-local DtN map, $T$. We point out that this map is also known as the Dirichlet-Neumann operator and the StekhlovPoincaré map. In Sect. 3 we will make a particular choice of $N$ which gives $T$ adjointness properties (see Appendix) that allow it to be computed in an accelerated fashion via the Operator Expansions algorithm (see Sect. 3.2).

\subsection{An $h p$-Finite Element Method}

In previous work [28], two of the authors investigated an algorithm which coupled an efficient implementation of the DtN map, $T$, on quite general $\mathcal{B}$ to a volumetric Finite Element discretization of (9) on $\Omega$. In that work, we used piecewise linear basis and test functions on triangles so that our approximate solution was a piecewise linear polynomial. The current work extends this idea to couple the DtN map to high-order pseudo-spectral schemes on unstructured nodal elements. In [34], Warburton, Pavarino, and Hesthaven demonstrated the flexibility, robustness, and exponential accuracy of these schemes and here we show that our DtN map fits seamlessly into this incarnation of the DtN-FE method. In brief, the method of Warburton, Pavarino, and Hesthaven begins with the classic variational formulation of (9) and a triangulation of $\Omega$ where, typically, the elements at the boundary must be curved. The solution is represented in a compact, element based fashion via Lagrange interpolating polynomials associated to carefully chosen nodes on each element. For the curved elements, an isoparametric mapping and Hall blending are utilized. We refer the interested reader to [34] for complete details. We note that this nodal pseudo-spectral scheme is able to realize the optimal order of accuracy, $(p+1)$, in the absence of curved elements, however, this rate drops to a suboptimal value, $p$, when curved elements are included.

An important issue in the numerical implementation of (9) is the well-posedness of the resulting discrete problem. In [29] two of the authors took up this question and showed rigorously that if the DtN map, $T$, is suitably modified (see $[9,15,17]$ ) then the discrete system is indeed well-posed. From a practical standpoint, however, we did not notice any instabilities in our numerical simulations using the unmodified DtN map and thus we advocate its use in generic computations.

\section{The Dirichlet-to-Neumann Map}

In order to completely specify the numerical method presented in the previous section we must compute the DtN map, $T$, at the Artificial Boundary $\mathcal{B}$. The case of a circular boundary

$$
\mathcal{B}=\{r=b\}
$$

has been investigated by Feng [10], Han and Wu [16], and Keller and Givoli [11-14, 20]. In this case the exact solution of (7) is

$$
w(r, \theta)=\sum_{p=-\infty}^{\infty} \frac{H_{p}^{(1)}(k r)}{H_{p}^{(1)}(k b)} \hat{\psi}_{p} e^{i p \theta},
$$

where $\hat{\psi}_{p}$ is the $p$-th Fourier coefficient of $\psi$ and $H_{p}^{(1)}$ is the $p$-th Hankel function of the first kind. From (8) the DtN map is

$$
T(r=b)[\psi]=-\sum_{p=-\infty}^{\infty} b k \frac{d_{z} H_{p}^{(1)}(k b)}{H_{p}^{(1)}(k b)} \hat{\psi}_{p} e^{i p \theta}=-k b \frac{d_{z} H_{D}^{(1)}(k b)}{H_{D}^{(1)}(k b)}[\psi]
$$


where $d_{z}$ is differentiation with respect the argument of the Hankel function, and we have identified the Fourier multiplier

$$
m(D)[\psi]:=\sum_{p=-\infty}^{\infty} m(p) \hat{\psi}_{p} e^{i p \theta} .
$$

This method was significantly expanded in [28] to include Artificial Boundaries of the form

$$
\mathcal{B}=\{r=b+g(\theta)\}
$$

i.e. perturbations of a circle. This generalization is significant as it allows the construction of Artificial Boundaries which are close to the surface of the scatterer, resulting in a computational domain, $\Omega$, with a modest number of elements. We will see evidence of this in Sect. 4 and show the remarkable gains that can be achieved over the original DtN-FE method with an appropriate choice of $\mathcal{B}$.

Of course the difficult task that remains is the computation of the DtN map on a domain with the perturbed shape (11). In fact, the form of our new class of Artificial Boundaries suggests that a perturbative method should be both convenient and highly accurate. To make this clearer, if we specify $g=\varepsilon f$, it can be shown [28] that both the field, $w=w(x, y ; \varepsilon)$, and the DtN map, $T=T(\varepsilon f)$, depend analytically upon $\varepsilon$ and that the expansions

$$
w(x, y ; \varepsilon)=\sum_{n=0}^{\infty} w_{n}(x, y) \varepsilon^{n}, \quad T(\varepsilon f)[\psi]=\sum_{n=0}^{\infty} T_{n}(f)[\psi] \varepsilon^{n},
$$

are strongly convergent in an appropriate Sobolev space. Clearly, a truncation of the Taylor series for the DtN map,

$$
T^{N}(\varepsilon f)[\psi]:=\sum_{n=0}^{N} T_{n}(f)[\psi] \varepsilon^{n}
$$

should prove to be a highly accurate approximation to $T$ and will be very convenient if a good formula for the $T_{n}$ can be identified. For this purpose several "Boundary Perturbation" techniques have been developed, and in the following two sections we describe two methods which are both fast and accurate.

\subsection{Field Expansions}

The method of "Field Expansions" (FE) for computational scattering can be traced back to the pioneering work of Rayleigh [21] and Rice [33], and was extended to a reliable highorder method by Bruno and Reitich [2-7]. This FE method is based upon the analyticity of $w$ and $T$, see (12), and recursion formulas for the $w_{n}$ and $T_{n}$ which can be realized with the following calculations for the case $\mathcal{B}=\{r=b+\varepsilon f\}$. We begin by inserting the expansion for $w,(12)$, into (7) and realizing that the $w_{n}$ must satisfy

$$
\begin{aligned}
& \Delta w_{n}+k^{2} w_{n}=0, \quad r>b, \\
& w_{n}(b, \theta)=\delta_{n, 0} \psi-\sum_{l=0}^{n-1} \frac{f^{n-l}}{(n-l) !} \partial_{r}^{n-l} w_{l}(b, \theta), \\
& \lim _{r \rightarrow \infty} r^{1 / 2}\left(\partial_{r} w_{n}-i k w_{n}\right)=0,
\end{aligned}
$$


where $\delta_{n, m}$ is the Kronecker delta. The solution of (14a) and (14c) is

$$
w_{n}(r, \theta)=\sum_{p=-\infty}^{\infty} d_{p, n} \frac{H_{p}^{(1)}(k r)}{H_{p}^{(1)}(k b)} e^{i p \theta} .
$$

Equation (14b) can be used to solve for $d_{p, n}$ :

$$
d_{p, n}=\delta_{n, 0} \hat{\psi}_{p}-\sum_{l=0}^{n-1} \sum_{q=-\infty}^{\infty} C_{p-q, n-l} \frac{d_{z}^{n-l} H_{q}^{(1)}(k b)}{H_{q}^{(1)}(k b)} d_{q, l}
$$

where $\hat{\psi}_{p}$ is the $p$ th Fourier coefficient of $\psi$,

$$
C_{l}(\theta):=\frac{k^{l} f^{l}(\theta)}{l !}, \quad C_{l}(\theta)=: \sum_{p=-\infty}^{\infty} C_{l, p} e^{i p \theta} .
$$

Given the $d_{p, n}$ we can now compute the $n$th term of the DtN map. At this point we define

$$
N_{g}:=\left(-(b+g), \partial_{\theta} g\right)^{T}
$$

as our normal vector, which will produce a DtN map with advantageous adjointness properties (see Sect. 3.2 and Appendix). Recalling (8) and (12), and $\nabla=\left(\partial_{r},(1 / r) \partial_{\theta}\right)^{T}$,

$$
\begin{aligned}
T(\varepsilon f)[\psi]= & \nabla w(b+\varepsilon f(\theta), \theta) \cdot N_{\varepsilon f} \\
= & \sum_{n=0}^{\infty} \sum_{p=-\infty}^{\infty}\left[-k(b+\varepsilon f) \frac{d_{z} H_{p}^{(1)}(k(b+\varepsilon f))}{H_{p}^{(1)}(k b)}\right. \\
& \left.+\frac{\varepsilon \partial_{\theta} f}{(b+\varepsilon f)}(i p) \frac{H_{p}^{(1)}(k(b+\varepsilon f))}{H_{p}^{(1)}(k b)}\right] d_{p, n} e^{i p \theta} \varepsilon^{n} .
\end{aligned}
$$

A recursion for the $T_{n}(f)$ can be realized by multiplying by $(b+\varepsilon f)$ (to remove quotients involving $\varepsilon$ ), and expanding the DtN map in powers of $\varepsilon$ :

$$
\begin{aligned}
T_{n}(f)[\psi]= & -k b \sum_{l=0}^{n} \sum_{p=-\infty}^{\infty} \frac{(k f)^{n-l}}{(n-l) !} \frac{d_{z}^{n+1-l} H_{p}^{(1)}(k b)}{H_{p}^{(1)}(k b)} d_{p, l} e^{i p \theta} \\
& -\frac{f}{b} T_{n-1}(f)[\psi] \\
& -2 k f \sum_{l=0}^{n-1} \sum_{p=-\infty}^{\infty} \frac{(k f)^{n-1-l}}{(n-1-l) !} \frac{d_{z}^{n-l} H_{p}^{(1)}(k b)}{H_{p}^{(1)}(k b)} d_{p, l} e^{i p \theta} \\
& -\frac{k}{b} f^{2} \sum_{l=0}^{n-2} \sum_{p=-\infty}^{\infty} \frac{(k f)^{n-2-l}}{(n-2-l) !} \frac{d_{z}^{n-1-l} H_{p}^{(1)}(k b)}{H_{p}^{(1)}(k b)} d_{p, l} e^{i p \theta} \\
& +\frac{1}{b}\left(\partial_{\theta} f\right) \sum_{l=0}^{n-1} \sum_{p=-\infty}^{\infty} \frac{(k f)^{n-1-l}}{(n-1-l) !} \frac{d_{z}^{n-1-l} H_{p}^{(1)}(k b)}{H_{p}^{(1)}(k b)}(i p) d_{p, l} e^{i p \theta}
\end{aligned}
$$


or,

$$
\begin{aligned}
T_{n}(f)[\psi]= & -k b \sum_{l=0}^{n} C_{n-l} \frac{d_{z}^{n+1-l} H_{D}^{(1)}(k b)}{H_{D}^{(1)}(k b)} \mathcal{D}_{l} \\
& -\frac{f}{b} T_{n-1}(f)[\psi] \\
& -2 k f \sum_{l=0}^{n-1} C_{n-1-l} \frac{d_{z}^{n-l} H_{D}^{(1)}(k b)}{H_{D}^{(1)}(k b)} \mathcal{D}_{l} \\
& -\frac{k}{b} f^{2} \sum_{l=0}^{n-2} C_{n-2-l} \frac{d_{z}^{n-1-l} H_{D}^{(1)}(k b)}{H_{p}^{(1)}(k b)} \mathcal{D}_{l} \\
& +\frac{1}{b}\left(\partial_{\theta} f\right) \sum_{l=0}^{n-1} C_{n-1-l} \frac{d_{z}^{n-1-l} H_{D}^{(1)}(k b)}{H_{D}^{(1)}(k b)} \partial_{\theta} \mathcal{D}_{l},
\end{aligned}
$$

where

$$
\mathcal{D}_{l}(\theta):=\sum_{p=-\infty}^{\infty} d_{p, l} e^{i p \theta}
$$

Once these are computed, the approximation $T^{N}$ (c.f. (13)) of $T$ can be implemented.

\subsection{Operator Expansions}

A second approach to the numerical simulation of DtN maps, in the setting of acoustic scattering, is due to Milder [22-27]. In this "Operator Expansions" (OE) method one works exclusively with the operator, $T$, and, using the analyticity properties of the DtN map and the expansion (12), this method computes the $T_{n}$ directly. We begin by noting that a solution of (7a) and (7c) is

$$
w_{p}(r, \theta)=H_{p}^{(1)}(k r) e^{i p \theta}
$$

Since

$$
\psi_{p}(\theta)=w_{p}(b+\varepsilon f, \theta)=H_{p}^{(1)}(k(b+\varepsilon f)) e^{i p \theta}
$$

the definition of the DtN map, (8), yields

$$
\begin{aligned}
T(\varepsilon f)\left[H_{p}^{(1)}(k(b+\varepsilon f)) e^{i p \theta}\right]= & -\left.(b+\varepsilon f) \partial_{r}\left(H_{p}^{(1)}(k r) e^{i p \theta}\right)\right|_{r=b+\varepsilon f} \\
& +\left.\frac{\varepsilon \partial_{\theta} f}{b+\varepsilon f} \partial_{\theta}\left(H_{p}^{(1)}(k r) e^{i p \theta}\right)\right|_{r=b+\varepsilon f},
\end{aligned}
$$

that is

$$
\begin{aligned}
T(\varepsilon f)\left[H_{p}^{(1)}(k(b+\varepsilon f)) e^{i p \theta}\right]= & -k(b+\varepsilon f) d_{z} H_{p}^{(1)}(k(b+\varepsilon f)) e^{i p \theta} \\
& +\frac{\varepsilon \partial_{\theta} f}{b+\varepsilon f}(i p) H_{p}^{(1)}(k(b+\varepsilon f)) e^{i p \theta}
\end{aligned}
$$


Expanding in powers of $\varepsilon$ and recognizing $(b+\varepsilon f)^{-1}$ as a geometric series,

$$
\begin{aligned}
& \left(\sum_{n=0}^{\infty} T_{n}(f) \varepsilon^{n}\right)\left[\sum_{n=0}^{\infty} d_{z}^{n} H_{p}^{(1)}(k b) \frac{k^{n} f^{n}}{n !} e^{i p \theta} \varepsilon^{n}\right] \\
& =-k b \sum_{n=0}^{\infty} d_{z}^{n+1} H_{p}^{(1)}(k b) \frac{k^{n} f^{n}}{n !} e^{i p \theta} \varepsilon^{n} \\
& \quad-k(\varepsilon f) \sum_{n=0}^{\infty} d_{z}^{n+1} H_{p}^{(1)}(k b) \frac{k^{n} f^{n}}{n !} e^{i p \theta} \varepsilon^{n} \\
& \quad+\varepsilon\left(\partial_{\theta} f\right)\left(\frac{1}{b} \sum_{n=0}^{\infty}\left(\frac{-f}{b}\right)^{n} \varepsilon^{n}\right)(i p)\left(\sum_{n=0}^{\infty} d_{z}^{n} H_{p}^{(1)}(k b) \frac{k^{n} f^{n}}{n !} e^{i p \theta} \varepsilon^{n}\right) .
\end{aligned}
$$

At order $\varepsilon^{0}$ we find

$$
T_{0}\left[H_{p}^{(1)}(k b) e^{i p \theta}\right]=-k b d_{z} H_{p}^{(1)}(k b) e^{i p \theta},
$$

which simplifies to

$$
T_{0}\left[e^{i p \theta}\right]=-k b \frac{d_{z} H_{p}^{(1)}(k b)}{H_{p}^{(1)}(k b)} e^{i p \theta} .
$$

Since any periodic function $\psi(x)$ can be expressed as a linear combination of complex $\operatorname{exponentials~} \exp (i p \theta)$ through their Fourier series, we can conclude that

$$
T_{0}[\psi]=-k b \frac{d_{z} H_{D}^{(1)}(k b)}{H_{D}^{(1)}(k b)}[\psi],
$$

which, of course, coincides with (10).

At order $n$ (18) yields, recalling that $C_{n}=k^{n} f^{n} / n$ !,

$$
\begin{aligned}
T_{n}(f)\left[e^{i p \theta}\right]= & -k b C_{n} \frac{d_{z}^{n+1} H_{p}^{(1)}(k b)}{H_{p}^{(1)}(k b)} e^{i p \theta}-k f C_{n-1} \frac{d_{z}^{n} H_{p}^{(1)}(k b)}{H_{p}^{(1)}(k b)} e^{i p \theta} \\
& +\left(\partial_{\theta} f\right) \sum_{l=0}^{n-1} C_{l} \frac{1}{b}\left(\frac{-f}{b}\right)^{l}(i p) \frac{d_{z}^{n-l-1} H_{p}^{(1)}(k b)}{H_{p}^{(1)}(k b)} e^{i p \theta} \\
& -\sum_{l=0}^{n-1} T_{l}(f)\left[C_{n-l} \frac{d_{z}^{n-l} H_{p}^{(1)}(k b)}{H_{p}^{(1)}(k b)} e^{i p \theta}\right]
\end{aligned}
$$

Again, recognizing $\psi$ as a sum of Fourier modes, $e^{i p \theta}$, and identifying factors such as (ip) with operators $\partial_{\theta}$, we write

$$
T_{n}(f)[\psi]=-b k C_{n} \frac{d_{z}^{n+1} H_{D}^{(1)}(k b)}{H_{D}^{(1)}(k b)}[\psi]-k f C_{n-1} \frac{d_{z}^{n} H_{D}^{(1)}(k b)}{H_{D}^{(1)}(k b)}[\psi]
$$




$$
\begin{aligned}
& +\sum_{l=0}^{n-1} C_{n-1-l} \frac{\left(\partial_{\theta} f\right)(-1)^{l} f^{l}}{b^{l+1}} \partial_{\theta} \frac{d_{z}^{n-1-l} H_{D}^{(1)}(k b)}{H_{D}^{(1)}(k b)}[\psi] \\
& -\sum_{l=0}^{n-1} T_{l}(f)\left[C_{n-l} \frac{d_{z}^{n-l} H_{D}^{(1)}(k b)}{H_{D}^{(1)}(k b)}[\psi]\right] .
\end{aligned}
$$

At this point a careful accounting of the computational complexity of this OE algorithm is in order. As a preliminary step, the powers of $C_{l}=k^{l} f^{l} / l$ ! can be computed in $\mathcal{O}\left(l N_{x} \log \left(N_{x}\right)\right)$ if $N_{x}$ Fourier coefficients for $f$ are retained. However, an inspection of the final term in (19) reveals that, given a fixed $\psi$, the cost of this algorithm grows factorially in $n$ as $T_{l}$ must be recomputed on a different argument $n$-many times at each order. This clearly compares very unfavorably with the $\mathcal{O}\left(n^{2} N_{x} \log \left(N_{x}\right)\right)$ cost of the FE algorithm. However, we show in Appendix that the adjointness properties of $T$ can be used to compute the term $T_{n}$ in $\mathcal{O}\left(n N_{x} \log \left(N_{x}\right)\right)$ yielding an overall cost identical to the FE method.

To conclude this section we mention that, in practice, there is rarely a reason to choose either the FE or OE algorithm over the other. As we showed in [31, 32], the performance of the algorithms is nearly identical over a wide range of problem configurations. However, it was discovered in $[31,32]$ that the ill-conditioning which can hamper these algorithms for very large and/or very rough deformations is a little stronger for the OE algorithm, and thus the FE approach may be preferable (though the comparative results we report in Tables 19 and 21 which show no appreciable difference, were representative of the experiments in this paper). Of course, in the case of very large and/or very rough boundaries a new approach may be necessary and we refer the interested reader to [32] for several possibilities.

\section{Numerical Results}

In this section we present the results of some numerical experiments which illustrate the flexibility and applicability of our new algorithm. Among the wide array of possible computations we could attempt, we have chosen three which are representative of the capabilities of our approach: Scattering from a cylinder, scattering from an ellipse, and scattering from a "star-shaped" obstacle. The first is chosen as we can appeal to an exact solution which will validate our numerical simulations, the second is a non-trivial generalization of the first, while the third demonstrates quite effectively how our new approach can enable huge computational savings with a well-chosen Artificial Boundary.

To give an idea of the performance of the numerical scheme, we have selected, for all of the simulations given below, two sample frequencies:

$$
k_{1}=60 \pi / 180 \approx 1.04719, \quad k_{2}=600 \pi / 180 \approx 10.4719 .
$$

These are simply two rather low-frequency values of $k$ which avoid any numerical resonances [29]. For convenience of presentation these are listed below as $k \approx 1$ and $k \approx 10$.

Before beginning we note that the coupling of our Non-Reflecting boundary condition to this FEM necessitates numerical quadratures to deliver approximate Fourier coefficients of basis functions to the DtN map $T$. These quadratures were computed with very high accuracy and produce negligible errors compared to those incurred by the rest of the scheme. Finally, for ease of comparison with our previous work [28], we again compute errors in the $L^{\infty}$ norm which is justified since solutions of elliptic PDE on regular domains result in smooth solutions. 


\subsection{Scattering from a Cylinder}

To begin we consider radiation incident upon a perfectly conducting scatterer shaped by a circle of radius one so that

$$
\Sigma_{c}=\{r<a\}, \quad \Gamma_{c}=\{r=a\}, \quad a=1,
$$

c.f. Sect. 2. This obstacle can be efficiently enclosed by a transparent boundary of circular shape with radius $b$,

$$
\mathcal{B}=\{r=b\}
$$

With this choice, we recover the DtN-FE method of Feng [10], Han and Wu [16], and Keller and Givoli [20]. However, in all of these implementations only a piecewise linear FEM was utilized. For the first time we present numerical results of this DtN-FE method coupled to a high-order $h p$-FEM. A convenient feature of this particular numerical example is the existence of an exact solution [8] for this configuration which we utilize in simulations presented below. The numerical parameters for the circular scatterer simulations are summarized in Table $1(b=2)$ and Table $6(b=1.2)$. As there is no perturbation of the Artificial Boundary we obtain an exact boundary condition at $\mathcal{B}$ with $N=0$ terms in the expansion of the DtN map; furthermore, both the $\mathrm{OE}$ and $\mathrm{FE}$ algorithms deliver the same expression.

In Tables 2-5 $(b=2)$ and 7-10 $(b=1.2)$ we present the results of our numerical simulations as compared to the exact solution. In these, and all future numerical results, $N_{f}$ is the number of Fourier coefficients retained in the approximation of $\xi$ (the Dirichlet data) and, if necessary, $f$ (the boundary perturbation). Additionally, $N_{e l}$ is the number of elements in the triangulation of the domain, while $N_{\Gamma}$ and $N_{\mathcal{B}}$ are the number of points on the scatterer and Artificial Boundary, respectively. In these tables we notice several things, first, our new boundary condition never interferes with the performance of the underlying FEM. To

Table 1 Mesh data for the circular scatterer $(a=1)$ with a circular Artificial Boundary $(b=2) . h_{1} \approx 0.25$ and $N_{e l}$ is the number of elements in the mesh. $N_{\Gamma}$ and $N_{\mathcal{B}}$ represent the number of points on the scatterer and Artificial Boundaries, respectively

Table 2 Circular scatterer $(a=1)$ with a far circular Artificial Boundary $(b=2)$ and scheme order $p=1$

\begin{tabular}{llrrr}
\hline Experiment & $h / h_{1}$ & \multicolumn{1}{c}{$N_{e l}$} & $N_{\Gamma}$ & $N_{\mathcal{B}}$ \\
\hline CC(1) & 1 & 302 & 24 & 46 \\
CC(2) & $1 / 2$ & 1208 & 48 & 92 \\
CC(3) & $1 / 4$ & 4832 & 96 & 184 \\
CC(4) & $1 / 8$ & 19328 & 192 & 368 \\
CC(5) & $1 / 16$ & 77312 & 384 & 736 \\
\hline
\end{tabular}

\begin{tabular}{llllll}
\hline$h / h_{1}$ & $k \approx 1$ & & & $k \approx 10$ & \\
\cline { 2 - 3 } \cline { 5 - 6 } & $N_{f}$ & $L^{\infty_{\text {-error }}}$ & & $N_{f}$ & $L^{\infty_{\text {-error }}}$ \\
\hline 1 & 12 & $3.74 \mathrm{E}-03$ & 32 & $1.63 \mathrm{E}+00$ \\
$1 / 2$ & 12 & $1.04 \mathrm{E}-03$ & 32 & $8.13 \mathrm{E}-01$ \\
$1 / 4$ & 12 & $3.00 \mathrm{E}-04$ & 32 & $2.01 \mathrm{E}-01$ \\
$1 / 8$ & 12 & $9.53 \mathrm{E}-05$ & 32 & $5.22 \mathrm{E}-02$ \\
$1 / 16$ & 12 & $3.00 \mathrm{E}-05$ & 32 & $1.33 \mathrm{E}-02$ \\
$q$ & 1.74 & & & & \\
\hline
\end{tabular}


Table 3 Circular scatterer $(a=1)$ with a far circular Artificial Boundary $(b=2)$ and scheme order $p=2$

Table 4 Circular scatterer $(a=1)$ with a far circular Artificial Boundary $(b=2)$ and scheme order $p=3$

Table 5 Circular scatterer $(a=1)$ with a far circular Artificial Boundary $(b=2)$ and scheme order $p=4$

\begin{tabular}{llllll}
\hline$h / h_{1}$ & $k \approx 1$ & & $k \approx 10$ & \\
\cline { 2 - 3 } \cline { 5 - 6 } & $N_{f}$ & $L^{\infty}$-error & & $N_{f}$ & $L^{\infty_{\text {-error }}}$ \\
\hline 1 & 12 & $3.64 \mathrm{E}-04$ & 36 & $2.72 \mathrm{E}-01$ \\
$1 / 2$ & 12 & $5.32 \mathrm{E}-05$ & 36 & $2.41 \mathrm{E}-02$ \\
$1 / 4$ & 12 & $7.28 \mathrm{E}-06$ & 36 & $2.12 \mathrm{E}-03$ \\
$1 / 8$ & 12 & $9.68 \mathrm{E}-07$ & 36 & $2.13 \mathrm{E}-04$ \\
$1 / 16$ & 14 & $1.24 \mathrm{E}-07$ & 36 & $2.28 \mathrm{E}-05$ \\
$q$ & 2.88 & & 3.39 & \\
\hline
\end{tabular}

\begin{tabular}{llllll}
\hline$h / h_{1}$ & \multicolumn{2}{l}{$k \approx 1$} & & $k \approx 10$ & \\
\cline { 2 - 3 } \cline { 5 - 6 } & $N_{f}$ & $L^{\infty}$-error & & $N_{f}$ & $L^{\infty}$-error \\
\hline 1 & 16 & $3.85 \mathrm{E}-05$ & 36 & $2.30 \mathrm{E}-02$ \\
$1 / 2$ & 16 & $2.95 \mathrm{E}-06$ & 36 & $1.59 \mathrm{E}-03$ \\
$1 / 4$ & 16 & $2.46 \mathrm{E}-07$ & 36 & $9.84 \mathrm{E}-05$ \\
$1 / 8$ & 16 & $1.96 \mathrm{E}-08$ & 38 & $6.36 \mathrm{E}-06$ \\
$1 / 16$ & 20 & $1.37 \mathrm{E}-09$ & & 40 & $4.01 \mathrm{E}-07$ \\
$q$ & 3.68 & & 3.96 & \\
\hline
\end{tabular}

\begin{tabular}{llllll}
\hline$h / h_{1}$ & $k \approx 1$ & & \multicolumn{2}{l}{$k \approx 10$} \\
\cline { 2 - 3 } \cline { 5 - 6 } & $N_{f}$ & $L^{\infty}$-error & & $N_{f}$ & $L^{\infty}$-error \\
\hline 1 & 16 & $3.12 \mathrm{E}-06$ & 36 & $3.87 \mathrm{E}-03$ \\
$1 / 2$ & 16 & $1.38 \mathrm{E}-07$ & 36 & $1.33 \mathrm{E}-04$ \\
$1 / 4$ & 18 & $4.79 \mathrm{E}-09$ & 38 & $5.88 \mathrm{E}-06$ \\
$1 / 8$ & 18 & $1.63 \mathrm{E}-10$ & 40 & $1.63 \mathrm{E}-07$ \\
$1 / 16$ & 20 & $5.29 \mathrm{E}-12$ & 40 & $2.50 \mathrm{E}-08$ \\
$q$ & 4.81 & & 4.42 & \\
\hline
\end{tabular}

Table 6 Mesh data for the circular scatterer $(a=1)$ with a circular Artificial Boundary $(b=1.2) . h_{1} \approx 0.25$ and $N_{e l}$ is the number of elements in the mesh. $N_{\Gamma}$ and $N_{\mathcal{B}}$ represent the number of points on the scatterer and Artificial Boundaries, respectively

\begin{tabular}{llrrr}
\hline Experiment & $h / h_{1}$ & \multicolumn{1}{c}{$N_{e l}$} & $N_{\Gamma}$ & $N_{\mathcal{B}}$ \\
\hline $\operatorname{CCC}(1)$ & 1 & 60 & 28 & 32 \\
$\operatorname{CCC}(2)$ & $1 / 2$ & 240 & 56 & 64 \\
$\operatorname{CCC}(3)$ & $1 / 4$ & 960 & 112 & 128 \\
$\operatorname{CCC}(4)$ & $1 / 8$ & 3840 & 224 & 256 \\
$\operatorname{CCC}(5)$ & $1 / 16$ & 15360 & 448 & 512 \\
\hline
\end{tabular}

make this more precise we compute, via a least-squares fit of the logarithm of the data, an approximation to $q$ in the error relationship

$$
e=C h^{q}
$$


Table 7 Circular scatterer $(a=1)$ with a near circular scheme order $p=1$ Artificial Boundary $(b=1.2)$ and

\begin{tabular}{|c|c|c|c|c|}
\hline \multirow[t]{2}{*}{$h / h_{1}$} & \multicolumn{2}{|c|}{$k \approx 1$} & \multicolumn{2}{|c|}{$k \approx 10$} \\
\hline & $N_{f}$ & $L^{\infty}$-error & $N_{f}$ & $L^{\infty}$-error \\
\hline 1 & 10 & $5.92 \mathrm{E}-03$ & 28 & $4.90 \mathrm{E}-01$ \\
\hline $1 / 2$ & 10 & $1.75 \mathrm{E}-03$ & 28 & $1.47 \mathrm{E}-01$ \\
\hline $1 / 4$ & 12 & $4.89 \mathrm{E}-04$ & 30 & $4.29 \mathrm{E}-02$ \\
\hline $1 / 8$ & 12 & $1.45 \mathrm{E}-04$ & 32 & $1.36 \mathrm{E}-02$ \\
\hline $1 / 16$ & 14 & $3.84 \mathrm{E}-05$ & 32 & $3.88 \mathrm{E}-03$ \\
\hline$q$ & 1.81 & & 1.74 & \\
\hline
\end{tabular}

Table 8 Circular scatterer $(a=1)$ with a near circular Artificial Boundary $(b=1.2)$ and scheme order $p=2$

\begin{tabular}{llllll}
\hline$h / h_{1}$ & $k \approx 1$ & & $k \approx 10$ & \\
\cline { 2 - 3 } \cline { 5 - 6 } & $N_{f}$ & $L^{\infty_{\text {-error }}}$ & & $N_{f}$ & $L^{\infty_{\text {-error }}}$ \\
\hline 1 & 12 & $5.32 \mathrm{E}-04$ & 30 & $1.11 \mathrm{E}-01$ \\
$1 / 2$ & 12 & $5.40 \mathrm{E}-05$ & 32 & $1.11 \mathrm{E}-02$ \\
$1 / 4$ & 14 & $7.13 \mathrm{E}-06$ & 34 & $1.60 \mathrm{E}-03$ \\
$1 / 8$ & 16 & $9.27 \mathrm{E}-07$ & 36 & $1.98 \mathrm{E}-04$ \\
$1 / 16$ & 16 & $1.39 \mathrm{E}-07$ & 38 & $2.97 \mathrm{E}-05$ \\
$q$ & 2.97 & & & \\
\hline
\end{tabular}

Table 9 Circular scatterer $(a=1)$ with a near circular Artificial Boundary $(b=1.2)$ and scheme order $p=3$

\begin{tabular}{llllll}
\hline$h / h_{1}$ & $k \approx 1$ & & & $k \approx 10$ & \\
\cline { 2 - 3 } \cline { 5 - 6 } & $N_{f}$ & $L^{\infty}$-error & & $N_{f}$ & $L^{\infty_{\text {-error }}}$ \\
\hline 1 & 14 & $2.92 \mathrm{E}-05$ & 34 & $1.35 \mathrm{E}-02$ \\
$1 / 2$ & 16 & $2.55 \mathrm{E}-06$ & 36 & $1.19 \mathrm{E}-03$ \\
$1 / 4$ & 18 & $1.83 \mathrm{E}-07$ & 38 & $8.50 \mathrm{E}-05$ \\
$1 / 8$ & 18 & $1.35 \mathrm{E}-08$ & 42 & $5.18 \mathrm{E}-06$ \\
$1 / 16$ & 20 & $9.53 \mathrm{E}-10$ & 44 & $3.80 \mathrm{E}-07$ \\
$q$ & 3.74 & & 3.81 & \\
\hline
\end{tabular}

We find that it is always within a small tolerance of the expected value specified by FEM theory.

The second thing to notice is that, in any of these experiments, with a fixed element size one can always realize a comparable approximation with far fewer elements (giving enormous computational savings) by choosing $b$ closer to $a(b=1.2$ versus $b=2)$, see Fig. 2. While this may, on the surface, seem to be obvious from the outset, it is noteworthy that the transparent boundary condition is unaffected by the complicated structure of the solution so near to the scattering surface.

\subsection{Scattering from an Ellipse}

We now consider a perfectly conducting scatterer shaped by an ellipse

$$
x=a_{1} \cos (\theta), \quad y=a_{2} \sin (\theta),
$$


Table 10 Circular scatterer $(a=1)$ with a near circular Artificial Boundary $(b=1.2)$ and scheme order $p=4$

\begin{tabular}{|c|c|c|c|c|}
\hline \multirow[t]{2}{*}{$h / h_{1}$} & \multicolumn{2}{|c|}{$k \approx 1$} & \multicolumn{2}{|c|}{$k \approx 10$} \\
\hline & $\overline{N_{f}}$ & $L^{\infty}$-error & $\overline{N_{f}}$ & $L^{\infty}$-error \\
\hline 1 & 16 & $2.13 \mathrm{E}-06$ & 38 & $2.40 \mathrm{E}-03$ \\
\hline $1 / 2$ & 18 & $7.76 \mathrm{E}-08$ & 40 & $1.01 \mathrm{E}-04$ \\
\hline $1 / 4$ & 20 & $2.99 \mathrm{E}-09$ & 44 & $3.17 \mathrm{E}-06$ \\
\hline $1 / 8$ & 20 & $1.03 \mathrm{E}-10$ & 46 & $1.04 \mathrm{E}-07$ \\
\hline $1 / 16$ & 22 & $3.88 \mathrm{E}-12$ & 50 & $3.29 \mathrm{E}-09$ \\
\hline$q$ & 4.77 & & 4.89 & \\
\hline
\end{tabular}

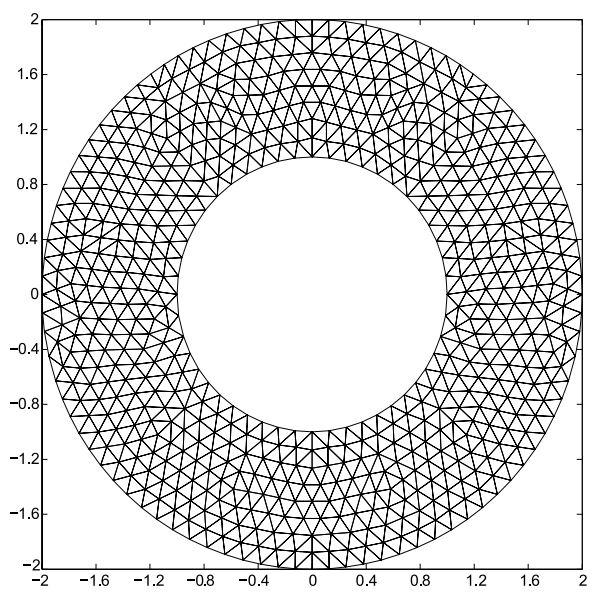

(a) $b=2$

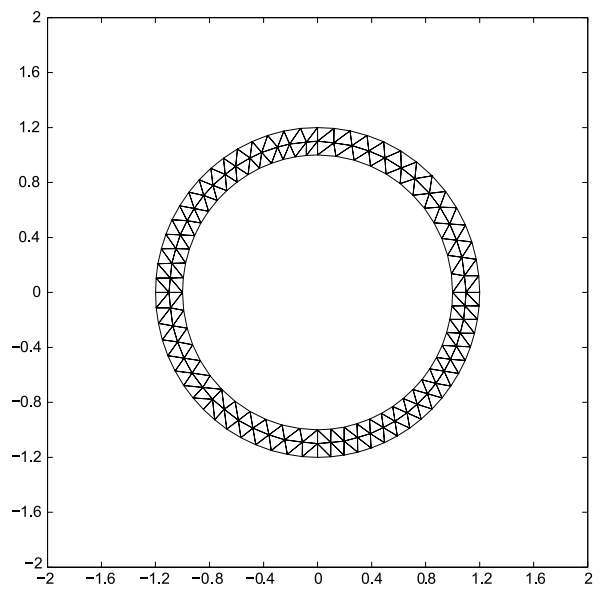

(b) $b=1.2$

Fig. 2 Computational meshes at refinement level $h / h_{1}=1 / 2\left(h_{1} \approx 0.25\right)$ for scattering from a circle with circular Artificial Boundaries. Left: $b=2$. Right: $b=1.2$

or, in polar coordinates,

$$
r=a_{1} \sqrt{1-e^{2} \sin ^{2}(\theta)}
$$

where $e=\sqrt{1-a_{2}^{2} / a_{1}^{2}}$ is the eccentricity. In this case

$$
\Sigma_{e}=\left\{r<a_{1} \sqrt{1-e^{2} \sin ^{2}(\theta)}\right\}, \quad \Gamma_{e}=\left\{r=a_{1} \sqrt{1-e^{2} \sin ^{2}(\theta)}\right\} .
$$

For this scatterer we display two sets of results, one where we enclose it with a circular Artificial Boundary,

$$
\mathcal{B}=\left\{r=b_{1}\right\}
$$

and a second where the ellipse is enclosed by another ellipse,

$$
\mathcal{B}=\left\{r=b_{1} \sqrt{1-\bar{e}^{2} \sin ^{2}(\theta)}\right\}
$$




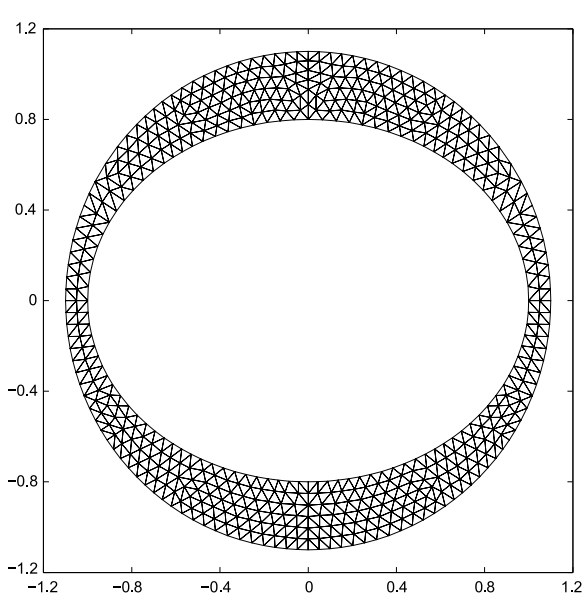

(a) Circular Artificial Boundary

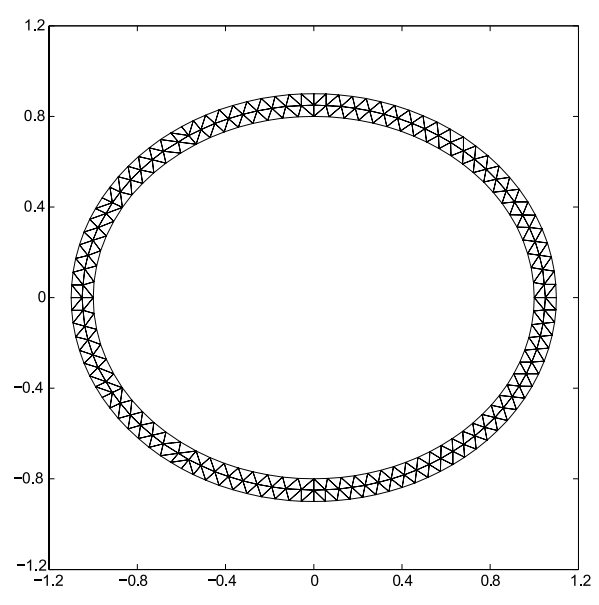

(b) Elliptical Artificial Boundary

Fig. 3 Computational meshes at refinement level $h / h_{1}=1 / 2\left(h_{1} \approx 0.25\right)$ for scattering from an ellipse. Left: Circular Artificial Boundary. Right: Elliptical Artificial Boundary

and $\bar{e}=\sqrt{1-b_{2}^{2} / b_{1}^{2}}$; see Fig. 3 .

The numerical parameters for these experiments are listed in Tables 11 and 16, and we point out that, in the latter case, an exact boundary condition at the Artificial Boundary, $\mathcal{B}$, would require an infinite number of terms in the expansion of the DtN map, (12). As we mentioned before, we approximate this infinite expansion with a truncated Taylor series, (13), where we have chosen $N=8$. An additional consideration in this case is how the partial sum (13) is to be computed. A strong case has been made in previous literature (see, e.g., $[3,7,30,32])$ for using Padé approximation [1] as it not only enhances convergence within the disk of analyticity of (12), but also allows for the summation of the series outside this disk. For the remainder of the experiments which require $N>0$ (i.e., $\mathcal{B}$ non-circular) we use Padé summation. Finally, in all of the results presented in this section, save the last (Table 21), we have used the OE recursions. In this final simulation we use the FE formulas with Padé summation to show that the results are nearly identical (compare Table 19 with Table 21).

The results for the elliptical scatterer and circular Artificial Boundary are displayed in Tables $12-15$, for polynomial orders $p=1,2,3,4$, respectively. Here, as there is no convenient exact solution, we measure the convergence of our solution via the Cauchy error:

$$
\left|u_{h_{n}}-u_{h_{n-1}}\right|_{L^{\infty}} .
$$

(Note that we cannot compute this quantity for $n=1$.) Again, we make a least-squares fit of the error data to the relationship $e=C h^{q}$ and, again, we see the order of accuracy that one expects from each polynomial order. Thus we conclude that the inclusion of the DtN map has no effect on the performance of our underlying FEM.

We remark briefly that measuring the Cauchy convergence of our numerical algorithm suffices for verification purposes as we have already presented thorough convergence tests of this method in [28]. Here the algorithm was not only tested (in the piecewise linear case, $p=1$ ) versus an exact "point-source" solution, but also compared against a highly resolved DtN-FE solution on a much larger domain. We direct the interested reader to Sect. 4.1 (particularly Table 1) and Sect. 4.2 of [28] for discussion and complete results. 
Table 11 Mesh data for the elliptical scatterer with $a_{1}=1$, $a_{2}=0.8$ and circular Artificial Boundary $b_{1}=1.1 . h_{1} \approx 0.25$ and $N_{e l}$ is the number of elements in the mesh. $N_{\Gamma}$ and $N_{\mathcal{B}}$ represent the number of points on the scatterer and Artificial Boundaries, respectively

Table 12 Elliptical scatterer $\left(a_{1}=1, a_{2}=0.8\right)$ with a circular Artificial Boundary $\left(b_{1}=1.1\right)$ and scheme order $p=1$

\begin{tabular}{llrrr}
\hline Experiment & $h / h_{1}$ & \multicolumn{1}{c}{$N_{e l}$} & $N_{\Gamma}$ & $N_{\mathcal{B}}$ \\
\hline EC(1) & 1 & 234 & 52 & 62 \\
EC(2) & $1 / 2$ & 936 & 104 & 124 \\
EC(3) & $1 / 4$ & 3744 & 208 & 248 \\
EC(4) & $1 / 8$ & 14976 & 416 & 496 \\
EC(5) & $1 / 16$ & 59904 & 832 & 992 \\
\hline
\end{tabular}

\begin{tabular}{llllll}
\hline$h / h_{1}$ & $k \approx 1$ & & & $k \approx 10$ & \\
\cline { 2 - 3 } & $N_{f}$ & $L^{\infty}$-error & & $N_{f}$ & $L^{\infty}$-error \\
\hline 1 & 4 & - & 24 & - \\
$1 / 2$ & 4 & $5.42 \mathrm{E}-03$ & 24 & $2.16 \mathrm{E}-01$ \\
$1 / 4$ & 4 & $1.68 \mathrm{E}-03$ & 24 & $8.10 \mathrm{E}-02$ \\
$1 / 8$ & 4 & $4.05 \mathrm{E}-04$ & 24 & $1.92 \mathrm{E}-02$ \\
$1 / 16$ & 4 & $1.13 \mathrm{E}-04$ & 24 & $6.51 \mathrm{E}-03$ \\
$q$ & 1.88 & & & \\
\hline
\end{tabular}

Table 13 Elliptical scatterer $\left(a_{1}=1, a_{2}=0.8\right)$ with a circular Artificial Boundary $\left(b_{1}=1.1\right)$ and scheme order $p=2$

\begin{tabular}{llllll}
\hline$h / h_{1}$ & $k \approx 1$ & & & $k \approx 10$ & \\
\cline { 2 - 3 } & $N_{f}$ & $L^{\infty_{\text {-error }}}$ & & $N_{f}$ & $L^{\infty_{\text {-error }}}$ \\
\hline 1 & 4 & - & 24 & - \\
$1 / 2$ & 4 & $3.07 \mathrm{E}-04$ & 24 & $4.77 \mathrm{E}-02$ \\
$1 / 4$ & 4 & $5.05 \mathrm{E}-05$ & 24 & $6.25 \mathrm{E}-03$ \\
$1 / 8$ & 4 & $6.95 \mathrm{E}-06$ & 24 & $8.59 \mathrm{E}-04$ \\
$1 / 16$ & 4 & $9.51 \mathrm{E}-07$ & 24 & $1.03 \mathrm{E}-04$ \\
$q$ & 2.79 & & & \\
\hline
\end{tabular}

Table 14 Elliptical scatterer $\left(a_{1}=1, a_{2}=0.8\right)$ with a circular Artificial Boundary $\left(b_{1}=1.1\right)$ and scheme order $p=3$

\begin{tabular}{|c|c|c|c|c|}
\hline \multirow[t]{2}{*}{$h / h_{1}$} & \multicolumn{2}{|c|}{$k \approx 1$} & \multicolumn{2}{|c|}{$k \approx 10$} \\
\hline & $N_{f}$ & $L^{\infty}$-error & $\overline{N_{f}}$ & $L^{\infty}$-error \\
\hline 1 & 4 & - & 24 & - \\
\hline $1 / 2$ & 4 & $1.48 \mathrm{E}-05$ & 24 & $1.86 \mathrm{E}-03$ \\
\hline $1 / 4$ & 4 & $1.38 \mathrm{E}-06$ & 24 & $2.36 \mathrm{E}-04$ \\
\hline $1 / 8$ & 4 & $8.62 \mathrm{E}-08$ & 24 & $1.35 \mathrm{E}-05$ \\
\hline $1 / 16$ & 4 & $6.23 \mathrm{E}-09$ & 24 & $9.97 \mathrm{E}-07$ \\
\hline$q$ & 3.76 & & 3.67 & \\
\hline
\end{tabular}

The results for the elliptical scatterer and elliptical Artificial Boundary are displayed in Tables 17-20, for polynomial orders $p=1,2,3,4$, respectively. Again, the order of accuracy that one would expect from each polynomial order is realized, however, we point out that accuracies quite comparable to the circular Artificial Boundary can be realized with the 
Table 15 Elliptical scatterer Artificial Boundary $\left(b_{1}=1.1\right)$ and scheme order $p=4$ $\left(a_{1}=1, a_{2}=0.8\right)$ with a circular

\begin{tabular}{|c|c|c|c|c|}
\hline \multirow[t]{2}{*}{$h / h_{1}$} & \multicolumn{2}{|c|}{$k \approx 1$} & \multicolumn{2}{|c|}{$k \approx 10$} \\
\hline & $N_{f}$ & $L^{\infty}$-error & $N_{f}$ & $L^{\infty}$-error \\
\hline 1 & 4 & - & 24 & - \\
\hline $1 / 2$ & 4 & $8.45 \mathrm{E}-07$ & 24 & $5.26 \mathrm{E}-04$ \\
\hline $1 / 4$ & 4 & $4.35 \mathrm{E}-08$ & 24 & $1.58 \mathrm{E}-05$ \\
\hline $1 / 8$ & 4 & $1.57 \mathrm{E}-09$ & 24 & $5.54 \mathrm{E}-07$ \\
\hline $1 / 16$ & 4 & $5.05 \mathrm{E}-11$ & 24 & $1.69 \mathrm{E}-08$ \\
\hline$q$ & 4.69 & & 4.96 & \\
\hline
\end{tabular}

\begin{tabular}{llrrr}
\hline Experiment & $h / h_{1}$ & \multicolumn{1}{c}{$N_{e l}$} & $N_{\Gamma}$ & $N_{\mathcal{B}}$ \\
\hline EE(1) & 1 & 94 & 43 & 51 \\
EE(2) & $1 / 2$ & 376 & 86 & 102 \\
EE(3) & $1 / 4$ & 1504 & 172 & 204 \\
EE(4) & $1 / 8$ & 6016 & 344 & 408 \\
EE(5) & $1 / 16$ & 24064 & 688 & 816 \\
\hline
\end{tabular}

\begin{tabular}{llllll}
\hline$h / h_{1}$ & $k \approx 1$ & & & $k \approx 10$ & \\
\cline { 2 - 3 } \cline { 5 - 5 } & $N_{f}$ & $L^{\infty}$-error & & $N_{f}$ & $L^{\infty_{\text {-error }}}$ \\
\hline 1 & 14 & - & 26 & - \\
$1 / 2$ & 14 & $4.06 \mathrm{E}-03$ & 26 & $2.64 \mathrm{E}-01$ \\
$1 / 4$ & 14 & $1.07 \mathrm{E}-03$ & 26 & $8.72 \mathrm{E}-02$ \\
$1 / 8$ & 14 & $2.98 \mathrm{E}-04$ & 26 & $2.47 \mathrm{E}-02$ \\
$1 / 16$ & 14 & $7.06 \mathrm{E}-05$ & 26 & $6.32 \mathrm{E}-03$ \\
$q$ & 1.94 & & & \\
\hline
\end{tabular}

Table 18 Elliptical scatterer $\left(a_{1}=1, a_{2}=0.8\right)$ with an elliptical Artificial Boundary $\left(b_{1}=1.1, b_{2}=0.9\right)$ and scheme order $p=2$

Table 17 Elliptical scatterer $\left(a_{1}=1, a_{2}=0.8\right)$ with an elliptical Artificial Boundary $\left(b_{1}=1.1, b_{2}=0.9\right)$ and scheme order $p=1$

Table 16 Mesh data for the 位ical scatterer with $a_{1}=1.0$ Boundary $b_{1}=1.1, b_{2}=0.9$. $h_{1} \approx 0.25$ and $N_{e l}$ is the number ofements in the mesh. $N_{\Gamma}$ and points on the scatterer and Artificial Boundaries respectively

\begin{tabular}{|c|c|c|c|c|}
\hline \multirow[t]{2}{*}{$h / h_{1}$} & \multicolumn{2}{|c|}{$k \approx 1$} & \multicolumn{2}{|c|}{$k \approx 10$} \\
\hline & $N_{f}$ & $L^{\infty}$-error & $\overline{N_{f}}$ & $L^{\infty}$-error \\
\hline 1 & 14 & - & 26 & - \\
\hline $1 / 2$ & 14 & $2.90 \mathrm{E}-04$ & 26 & $2.75 \mathrm{E}-02$ \\
\hline $1 / 4$ & 14 & $4.47 \mathrm{E}-05$ & 26 & $3.76 \mathrm{E}-03$ \\
\hline $1 / 8$ & 14 & $6.66 \mathrm{E}-06$ & 26 & $4.77 \mathrm{E}-04$ \\
\hline $1 / 16$ & 14 & $8.55 \mathrm{E}-07$ & 26 & $6.13 \mathrm{E}-05$ \\
\hline$q$ & 2.80 & & 2.94 & \\
\hline
\end{tabular}

elliptical $\mathcal{B}$ with the same element size but one half to one third the number of elements (see Tables 11 and 16). Again, we see the enormous computational savings that can be realized with our new approach. 
Table 19 Elliptical scatterer $\left(a_{1}=1, a_{2}=0.8\right)$ with an elliptical Artificial Boundary $\left(b_{1}=1.1, b_{2}=0.9\right)$ and scheme order $p=3$

\begin{tabular}{|c|c|c|c|c|}
\hline \multirow[t]{2}{*}{$h / h_{1}$} & \multicolumn{2}{|c|}{$k \approx 1$} & \multicolumn{2}{|c|}{$k \approx 10$} \\
\hline & $\overline{N_{f}}$ & $L^{\infty}$-error & $\overline{N_{f}}$ & $L^{\infty}$-error \\
\hline 1 & 14 & - & 26 & - \\
\hline $1 / 2$ & 14 & $1.47 \mathrm{E}-05$ & 26 & $3.28 \mathrm{E}-03$ \\
\hline $1 / 4$ & 14 & $9.45 \mathrm{E}-07$ & 26 & $2.45 \mathrm{E}-04$ \\
\hline $1 / 8$ & 14 & $6.47 \mathrm{E}-08$ & 26 & $1.29 \mathrm{E}-05$ \\
\hline $1 / 16$ & 14 & $4.72 \mathrm{E}-09$ & 26 & $8.56 \mathrm{E}-07$ \\
\hline$q$ & 3.87 & & 4.00 & \\
\hline
\end{tabular}

\begin{tabular}{|c|c|c|c|c|}
\hline \multirow[t]{2}{*}{$h / h_{1}$} & \multicolumn{2}{|c|}{$k \approx 1$} & \multicolumn{2}{|c|}{$k \approx 10$} \\
\hline & $N_{f}$ & $L^{\infty}$-error & $N_{f}$ & $L^{\infty}$-error \\
\hline 1 & 14 & - & 26 & - \\
\hline $1 / 2$ & 14 & $1.39 \mathrm{E}-06$ & 26 & $2.18 \mathrm{E}-04$ \\
\hline $1 / 4$ & 14 & $1.85 \mathrm{E}-07$ & 26 & $6.29 \mathrm{E}-06$ \\
\hline $1 / 8$ & 14 & $1.83 \mathrm{E}-09$ & 26 & $2.20 \mathrm{E}-07$ \\
\hline $1 / 16$ & 14 & $2.32 \mathrm{E}-10$ & 26 & $7.36 \mathrm{E}-09$ \\
\hline$q$ & 4.43 & & 4.94 & \\
\hline
\end{tabular}

\begin{tabular}{llllll}
\hline$h / h_{1}$ & $k \approx 1$ & & \multicolumn{2}{l}{$k \approx 10$} \\
\cline { 2 - 3 } \cline { 5 - 6 } & $N_{f}$ & $L^{\infty}$-error & & $N_{f}$ & $L^{\infty}$-error \\
\hline 1 & 14 & - & 26 & - \\
$1 / 2$ & 14 & $1.47 \mathrm{E}-05$ & 26 & $3.28 \mathrm{E}-03$ \\
$1 / 4$ & 14 & $9.45 \mathrm{E}-07$ & 26 & $2.45 \mathrm{E}-04$ \\
$1 / 8$ & 14 & $6.47 \mathrm{E}-08$ & 26 & $1.29 \mathrm{E}-05$ \\
$1 / 16$ & 14 & $4.72 \mathrm{E}-09$ & 26 & $8.56 \mathrm{E}-07$ \\
$q$ & 3.87 & & 4.00 & \\
\hline
\end{tabular}

Table 20 Elliptical scatterer $\left(a_{1}=1, a_{2}=0.8\right)$ with an elliptical Artificial Boundary $\left(b_{1}=1.1, b_{2}=0.9\right)$ and scheme order $p=4$
Table 21 Elliptical scatterer $\left(a_{1}=1, a_{2}=0.8\right)$ with an elliptical Artificial Boundary $\left(b_{1}=1.1, b_{2}=0.9\right)$ and scheme order $p=3$. FE recursions are used to compute DtN map terms $T_{n}$ (c.f. Table 19)

An important point to make regarding these simulations is that since an ellipse is a "separable" geometry one could implement a DtN map of the form (10) based upon the appropriate eigenfunctions for this geometry (in two dimensions the Mathieu functions [15]). Our new approach presents a significant improvement upon this idea since no additional coding is required: An ellipse is easily expressed as an analytic perturbation of a circle for which our DtN map, $T(\varepsilon)$, will converge extremely rapidly.

To close, we revisit the experiment presented in Table 19, i.e. scattering from an ellipse enclosed by an elliptical Artificial Boundary with polynomial order $p=3$. However, we now choose to compute the terms $T_{n}$ in the DtN map via the FE algorithm. As we can see from Table 21, the results are identical up to the accuracy of our tables. Indeed, this has been our general experience and we can advocate the use of either OE or FE for these low-frequency applications. 


\subsection{Scattering from a Star-Shaped Obstacle}

We now consider a scatterer shaped by a perturbation of a circle of radius one

$$
\Sigma_{s}=\{r<a+\delta \cos (4 \theta)\}, \quad \Gamma_{s}=\{r=a+\delta \cos (4 \theta)\}, \quad a=1, \quad \delta=0.4,
$$

which we term "star-shaped." In our first set of experiments with this geometry we consider the DtN-FE method which encloses $\Sigma_{s}$ with a circular Artificial Boundary

$$
\mathcal{B}=\{r=b\}
$$

where we have chosen $b=1.5$; see Fig. 4(a).

The numerical parameters for these simulations are given in Table 22, and the results of our experiments are summarized in Tables 23-26, for polynomial orders $p=1,2,3,4$, respectively. We note that the final refinements in Tables 25 and 26 are missing. This is due to the enormous size of these computations which necessitate the use of an iterative linear solver. With a desire to exclude the added considerations one must make for the convergence properties of such a scheme, and since these final data points are very unlikely to change our overall conclusions, we have omitted them.

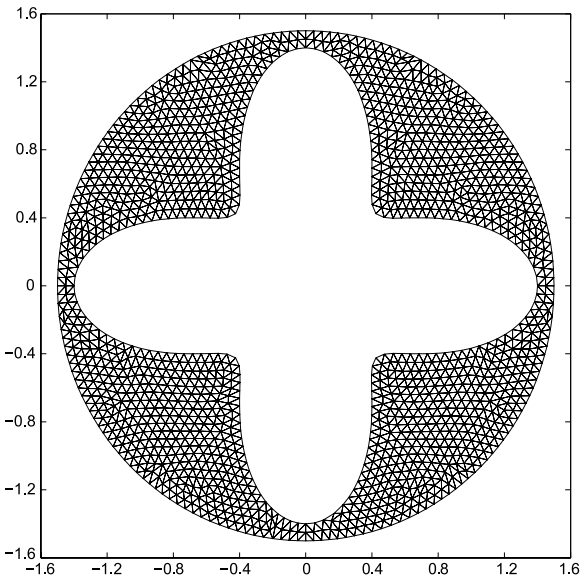

(a) Circular Artificial Boundary

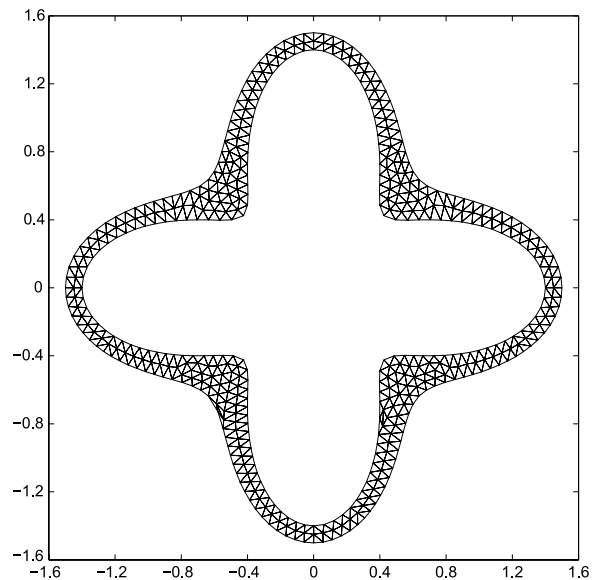

(b) Star-Shaped Artificial Boundary

Fig. 4 Computational meshes at refinement level $h / h_{1}=1 / 2\left(h_{1} \approx 0.25\right)$ for scattering from a star-shaped obstacle. Left: Circular Artificial Boundary. Right: Star-Shaped Artificial Boundary

Table 22 Mesh data for the star-shaped scatterer $(a=1$, $\delta=0.4)$ with a circular Artificial Boundary $(b=1.5) . h_{1} \approx 0.25$ and $N_{e l}$ is the number of elements in the mesh. $N_{\Gamma}$ and $N_{\mathcal{B}}$ represent the number of points on the scatterer and Artificial Boundaries, respectively

\begin{tabular}{llrrr}
\hline Experiment & $h / h_{1}$ & \multicolumn{1}{c}{$N_{e l}$} & \multicolumn{1}{c}{$N_{\Gamma}$} & $N_{\mathcal{B}}$ \\
\hline $\mathrm{SC}(1)$ & 1 & 717 & 89 & 88 \\
$\mathrm{SC}(2)$ & $1 / 2$ & 2868 & 178 & 176 \\
$\mathrm{SC}(3)$ & $1 / 4$ & 11472 & 356 & 352 \\
$\mathrm{SC}(4)$ & $1 / 8$ & 45888 & 712 & 704 \\
$\mathrm{SC}(5)$ & $1 / 16$ & 183552 & 1424 & 1408 \\
\hline
\end{tabular}


Table 23 Star-shaped scatterer ( $a=1, \delta=0.4$ ) with a circular Artificial Boundary $(b=1.5)$ and scheme order $p=1$

Table 24 Star-shaped scatterer $(a=1, \delta=0.4)$ with a circular Artificial Boundary $(b=1.5)$ and scheme order $p=2$

Table 25 Star-shaped scatterer $(a=1, \delta=0.4)$ with a circular Artificial Boundary $(b=1.5)$ and scheme order $p=3$

\begin{tabular}{llllll}
\hline$h / h_{1}$ & $k \approx 1$ & & & $k \approx 10$ & \\
\cline { 2 - 3 } \cline { 5 - 6 } & $N_{f}$ & $L^{\infty}$-error & & $N_{f}$ & $L^{\infty_{\text {-error }}}$ \\
\hline 1 & 4 & - & 32 & - \\
$1 / 2$ & 4 & $9.57 \mathrm{E}-03$ & 32 & $3.47 \mathrm{E}-01$ \\
$1 / 4$ & 4 & $2.10 \mathrm{E}-03$ & 32 & $1.14 \mathrm{E}-01$ \\
$1 / 8$ & 4 & $5.74 \mathrm{E}-04$ & 32 & $2.97 \mathrm{E}-02$ \\
$1 / 16$ & 4 & $1.44 \mathrm{E}-04$ & 32 & $7.30 \mathrm{E}-03$ \\
$q$ & 2.00 & & & \\
\hline
\end{tabular}

\begin{tabular}{|c|c|c|c|c|}
\hline \multirow[t]{2}{*}{$h / h_{1}$} & \multicolumn{2}{|c|}{$k \approx 1$} & \multicolumn{2}{|c|}{$k \approx 10$} \\
\hline & $N_{f}$ & $L^{\infty}$-error & $\overline{N_{f}}$ & $L^{\infty}$-error \\
\hline 1 & 4 & - & 32 & - \\
\hline $1 / 2$ & 4 & $5.66 \mathrm{E}-04$ & 32 & $2.25 \mathrm{E}-02$ \\
\hline $1 / 4$ & 4 & $9.59 \mathrm{E}-05$ & 32 & $2.86 \mathrm{E}-03$ \\
\hline $1 / 8$ & 4 & $1.36 \mathrm{E}-05$ & 32 & $3.53 \mathrm{E}-04$ \\
\hline $1 / 16$ & 4 & $1.90 \mathrm{E}-06$ & 32 & $4.29 \mathrm{E}-05$ \\
\hline$q$ & 2.75 & & 3.01 & \\
\hline
\end{tabular}

\begin{tabular}{llllll}
\hline$h / h_{1}$ & \multicolumn{2}{l}{$k \approx 1$} & & $k \approx 10$ & \\
\cline { 2 - 3 } \cline { 5 - 6 } & $N_{f}$ & $L^{\infty_{\text {-error }}}$ & & $N_{f}$ & $L^{\infty_{\text {-error }}}$ \\
\hline 1 & 4 & - & 32 & - \\
$1 / 2$ & 4 & $5.65 \mathrm{E}-05$ & 32 & $2.18 \mathrm{E}-03$ \\
$1 / 4$ & 4 & $4.52 \mathrm{E}-06$ & 32 & $1.61 \mathrm{E}-04$ \\
$1 / 8$ & 4 & $2.32 \mathrm{E}-07$ & 32 & $1.01 \mathrm{E}-05$ \\
$q$ & 3.96 & & 3.88 & \\
\hline
\end{tabular}

Table 26 Star-shaped scatterer ( $a=1, \delta=0.4$ ) with a circular Artificial Boundary $(b=1.5)$ and scheme order $p=4$

\begin{tabular}{llllll}
\hline$h / h_{1}$ & \multicolumn{2}{l}{$k \approx 1$} & & $k \approx 10$ \\
\cline { 2 - 3 } \cline { 5 - 6 } & $N_{f}$ & $L^{\infty}$-error & & $N_{f}$ & $L^{\infty}$-error \\
\hline 1 & 4 & - & 32 & - \\
$1 / 2$ & 4 & $4.44 \mathrm{E}-06$ & 32 & $2.26 \mathrm{E}-04$ \\
$1 / 4$ & 4 & $2.59 \mathrm{E}-07$ & 32 & $1.01 \mathrm{E}-05$ \\
$1 / 8$ & 4 & $7.33 \mathrm{E}-09$ & 32 & $2.87 \mathrm{E}-07$ \\
$q$ & 4.62 & & & 4.81 & \\
\hline
\end{tabular}

Next, we consider our new method with an Artificial Boundary shaped to mimic that of the scatterer, i.e.

$$
\mathcal{B}=\left\{r<b+\delta_{b} \cos (4 \theta)\right\}, \quad b=1.2, \quad \delta_{b}=0.3 ;
$$


Table 27 Mesh data for the star-shaped scatterer ( $a=1$, $\delta=0.4)$ with a star-shaped

Artificial Boundary ( $a=1.2$, $\left.\delta_{b}=0.3\right) . h_{1} \approx 0.25$ and $N_{e l}$ is the number of elements in the mesh. $N_{\Gamma}$ and $N_{\mathcal{B}}$ represent the number of points on the scatterer and Artificial Boundaries, respectively

Table 28 Star-shaped scatterer $(a=1, \delta=0.4)$ with a star-shaped Artificial Boundary $\left(b=1.2, \delta_{b}=0.3\right)$ and scheme order $p=1$

\begin{tabular}{llrrr}
\hline Experiment & $h / h_{1}$ & \multicolumn{1}{c}{$N_{e l}$} & \multicolumn{1}{c}{$N_{\Gamma}$} & $N_{\mathcal{B}}$ \\
\hline SS(1) & 1 & 189 & 78 & 75 \\
SS(2) & $1 / 2$ & 756 & 156 & 150 \\
SS(3) & $1 / 4$ & 3024 & 312 & 300 \\
SS $(4)$ & $1 / 8$ & 12096 & 624 & 600 \\
SS(5) & $1 / 16$ & 48384 & 1248 & 1200 \\
\hline
\end{tabular}

Table 29 Star-shaped scatterer $(a=1, \delta=0.4)$ with a star-shaped Artificial Boundary $\left(b=1.2, \delta_{b}=0.3\right)$ and scheme order $p=2$

\begin{tabular}{llllll}
\hline$h / h_{1}$ & $k \approx 1$ & & & $k \approx 10$ & \\
\cline { 2 - 3 } \cline { 5 - 6 } & $N_{f}$ & $L^{\infty}$-error & & $N_{f}$ & $L^{\infty}$-error \\
\hline 1 & 60 & - & 84 & - \\
$1 / 2$ & 60 & $1.12 \mathrm{E}-02$ & 84 & $4.18 \mathrm{E}-01$ \\
$1 / 4$ & 60 & $3.27 \mathrm{E}-03$ & 84 & $1.10 \mathrm{E}-01$ \\
$1 / 8$ & 60 & $8.82 \mathrm{E}-04$ & 84 & $3.19 \mathrm{E}-02$ \\
$1 / 16$ & 60 & $2.31 \mathrm{E}-04$ & 84 & $8.09 \mathrm{E}-03$ \\
$q$ & 1.87 & & & \\
\hline
\end{tabular}

\begin{tabular}{llllll}
\hline$h / h_{1}$ & $k \approx 1$ & & \multicolumn{2}{l}{$k \approx 10$} \\
\cline { 2 - 3 } \cline { 5 - 6 } & $N_{f}$ & $L^{\infty}$-error & & $N_{f}$ & $L^{\infty}$-error \\
\hline 1 & 60 & - & 84 & - \\
$1 / 2$ & 60 & $1.74 \mathrm{E}-03$ & 84 & $3.37 \mathrm{E}-02$ \\
$1 / 4$ & 60 & $2.64 \mathrm{E}-04$ & 84 & $4.55 \mathrm{E}-03$ \\
$1 / 8$ & 60 & $4.22 \mathrm{E}-05$ & 84 & $5.40 \mathrm{E}-04$ \\
$1 / 16$ & 60 & $4.86 \mathrm{E}-06$ & 84 & $7.23 \mathrm{E}-05$ \\
$q$ & 2.81 & & 2.97 & \\
\hline
\end{tabular}

see Fig. 4(b). For this geometry, the numerical parameters are given in Table 27. As in the case of the elliptical Artificial Boundary in Sect. 4.2, we must use the approximation (13) of the full DtN map to enforce our boundary condition. For this we use $N=8$, Padé summation, and the $\mathrm{OE}$ algorithm. The results with this star-shaped Artificial Boundary are summarized in Tables 28-31, for polynomial orders $p=1,2,3$, 4, respectively. We again notice the negligible impact that our Enhanced DtN-FE algorithm has on the convergence of the underlying FEM. An inspection of Tables 23-26 and Tables 28-31 once again indicates that the transparent boundary condition has no affect on the rate of convergence of the FEM, for any polynomial order.

\subsection{Computational Savings}

To conclude this section on numerical results, we would like to point out, quite explicitly, the enormous computational savings that can be realized by our methods. As with other implementations of the DtN-FE method, the total cost of a simulation is broken up into two parts: matrix assembly and the linear solve. The size of the relevant system is $\mathcal{O}\left(N_{e l}\right)$ with a 
Table 30 Star-shaped scatterer $(a=1, \delta=0.4)$ with a star-shaped Artificial Boundary $\left(b=1.2, \delta_{b}=0.3\right)$ and scheme order $p=3$

\begin{tabular}{|c|c|c|c|c|}
\hline \multirow[t]{2}{*}{$h / h_{1}$} & \multicolumn{2}{|c|}{$k \approx 1$} & \multicolumn{2}{|c|}{$k \approx 10$} \\
\hline & $\overline{N_{f}}$ & $L^{\infty}$-error & $\overline{N_{f}}$ & $L^{\infty}$-error \\
\hline 1 & 60 & - & 84 & - \\
\hline $1 / 2$ & 60 & $1.81 \mathrm{E}-04$ & 84 & $3.58 \mathrm{E}-03$ \\
\hline $1 / 4$ & 60 & $1.36 \mathrm{E}-05$ & 84 & $2.30 \mathrm{E}-04$ \\
\hline $1 / 8$ & 60 & $8.66 \mathrm{E}-07$ & 84 & $1.64 \mathrm{E}-05$ \\
\hline $1 / 16$ & 60 & $5.54 \mathrm{E}-08$ & 84 & $8.98 \mathrm{E}-07$ \\
\hline$q$ & 3.90 & & 3.97 & \\
\hline
\end{tabular}

\begin{tabular}{llllll}
\hline$h / h_{1}$ & $k \approx 1$ & & \multicolumn{2}{l}{$k \approx 10$} & \\
\cline { 2 - 3 } \cline { 5 - 6 } & $N_{f}$ & $L^{\infty}$-error & & $N_{f}$ & $L^{\infty}$-error \\
\hline 1 & 60 & - & 88 & - \\
$1 / 2$ & 60 & $2.19 \mathrm{E}-05$ & 88 & $4.11 \mathrm{E}-04$ \\
$1 / 4$ & 60 & $1.21 \mathrm{E}-06$ & 88 & $1.94 \mathrm{E}-05$ \\
$1 / 8$ & 60 & $3.50 \mathrm{E}-08$ & 88 & $6.57 \mathrm{E}-07$ \\
$1 / 16$ & 60 & $1.18 \mathrm{E}-09$ & 88 & $2.05 \mathrm{E}-08$ \\
$q$ & 4.77 & & 4.78 & \\
\hline
\end{tabular}

Table 31 Star-shaped scatterer $(a=1, \delta=0.4)$ with a star-shaped Artificial Boundary $\left(b=1.2, \delta_{b}=0.3\right)$ and scheme order $p=4$

dense sub-block of size $\mathcal{O}\left(N_{\mathcal{B}}\right)$. For the classical DtN-FE method, the sub-block can be assembled in time $\mathcal{O}\left(N_{\mathcal{B}} N_{f} \log \left(N_{f}\right)\right)$, while our new algorithm requires $\mathcal{O}\left(N_{\mathcal{B}} N_{f} \log \left(N_{f}\right) N\right)$ where $N \approx 8$. While this may appear to be a substantial increase in computational effort we note two things: first of all, this is a preprocessing step and only needs to be completed once if multiple simulations are desired and/or an iterative method is used. Second, it is typically the case that $N_{\mathcal{B}} / N_{e l}$ is in the range $1 / 50$ to $1 / 100$ so that even an eight-fold increase in the cost of the assembly of this sub-block will be negligible when compared to the total assembly cost.

More importantly to the total cost of the algorithm, once the matrix has been formed the cost of a direct linear solve (which we were able to use for all of the computations presented above) is $\mathcal{O}\left(N_{e l}^{3}\right)$, which, for large $N_{e l}$, will dominate the computational complexity. To quantify the savings that our new method enables, we consider the best error realized (for the case $k \approx 10$ ) by the classical DtN-FE method for scattering by the star-shaped obstacle. This best error is $2.87 \times 10^{-7}$, see Table 26 , with $p=4$ and $h / h_{1}=1 / 8$ implying, from Table 22, that $N_{e l}=45,888$ and $N_{\mathcal{B}}=704$. By contrast, with our new Enhanced DtN-FE method (where the star-shaped scatterer is enclosed by a star-shaped Artificial Boundary) we can realize a comparable error of $6.57 \times 10^{-7}$, see Table 31 , with $p=4$ and $h / h_{1}=1 / 8$ which, from Table 27, used $N_{e l}=12,096$ and $N_{\mathcal{B}}=600$. While the number of boundary points remains roughly constant, indicating an eight-fold increase in assembly time for the dense sub-block (but not the entire matrix), the number of elements can be reduced by a factor of 45,888/12,096 $\approx 3.79$ which indicates a speed-up in the linear solve by a factor of $3.79^{3} \approx 54.44$. We find this to be particularly compelling evidence for the utility of our new approach.

Remark 4.1 At this point one can ask what, if any, limitations there are to our approach. A careful inspection of our formulation, (9), reveals that the only true difficulty lies with the 
computation of the DtN map, $T(\mathcal{B})$. In this paper we have outlined a Boundary Perturbation (BP) approach to simulating the DtN map which has its shortcomings (given subsequently), however, this is not the only avenue that one may pursue and another method (e.g., one based upon Integral Equations) may yield much better results. Regarding our BP methodology, the first restriction is that the transparent boundary be expressible as $r=b+g(\theta)$, i.e. the graph of a function in the $\theta$ variable. As we have seen, this does not mandate that the truncated domain, $\Omega$, be convex, however, it does impose some restrictions. Additionally, the analyticity theorem for the DtN map [28] states that the boundary perturbation, $f$, be sufficiently smooth, and that the perturbation size, $\varepsilon$, be sufficiently small. In theory this not very restrictive: $f$ Lipschitz can be accommodated [18] and $\varepsilon$ can be any real number (up to physical obstruction, e.g. $\varepsilon<b$ in this configuration). However, in practice, to realize a well-resolved solution for a large and/or irregular obstacle, one is typically forced to choose a large number $N$ of terms in the Taylor series expansion of the DtN map. In this paper we have striven to choose boundaries which give rise to numerical simulations where $N$ may be chosen fairly small $(N=8)$. However, if one is willing to utilize 20,30 , or more terms then much more irregular artificial boundaries can be used.

\section{Conclusions}

In this paper we have demonstrated not only how the DtN-FE method can be correctly combined with a high-order $h p$-FEM to yield numerical results which do not degrade the performance of the underlying FEM, but also how the fundamental Artificial Boundary shape requirement can be relaxed to allow for quite general shapes. This "Enhanced DtN-FE Method" has been shown to be quite robust and flexible, and, with a suitably chosen Artificial Boundary, can deliver accurate answers with one half to one quarter the number of finite elements required by the DtN-FE method.

Acknowledgements DPN gratefully acknowledges support from the NSF through grant No. DMS0537511 .

\section{Appendix: Adjointness of the Dirichlet-to-Neumann Map}

In this appendix we describe the adjointness properties of the DtN map, $T$, which enable its rapid evaluation in the "Operator Expansions" (OE) methodology (see Sect. 3.2). To begin, let us study the adjointness of Fourier multipliers with the following Lemma.

Lemma A.1 If the Fourier multiplier $m(D)$ has symbol $m(p)$ then its adjoint $n(D):=$ $m^{*}(D)$ has symbol $n(p)=\overline{m(p)}$.

Proof We begin by recalling that the $L^{2}$-inner product for complex-valued, $2 \pi$-periodic functions $\xi$ and $\psi$ satisfies

$$
\begin{aligned}
\langle\xi, \psi\rangle & =\int_{0}^{2 \pi} \xi(\theta) \overline{\psi(\theta)} d \theta=\int_{0}^{2 \pi}\left(\sum_{p=-\infty}^{\infty} \hat{\xi}_{p} e^{i p \theta}\right) \overline{\left(\sum_{p=-\infty}^{\infty} \hat{\psi}_{p} e^{i p \theta}\right)} d \theta \\
& =\sum_{p=-\infty}^{\infty} \sum_{q=-\infty}^{\infty} \hat{\xi}_{p} \overline{\hat{\psi}_{q}} \int_{0}^{2 \pi} e^{i(p-q) \theta} d \theta=2 \pi \sum_{p=-\infty}^{\infty} \hat{\xi}_{p} \overline{\hat{\psi}_{p}}
\end{aligned}
$$


With this calculation in mind we can now compute:

$$
\begin{aligned}
\langle m(D) \xi, \psi\rangle & =2 \pi \sum_{p=-\infty}^{\infty} m(p) \hat{\xi}_{p} \overline{\hat{\psi}_{p}}=2 \pi \sum_{p=-\infty}^{\infty} \hat{\xi}_{p} \overline{\overline{m(p)} \hat{\psi}_{p}} \\
& =2 \pi \sum_{p=-\infty}^{\infty} \hat{\xi}_{p} \overline{n(p) \hat{\psi}_{p}}=\langle\xi, n(D) \psi\rangle
\end{aligned}
$$

so that $m^{*}=n$ where $n(p)=\overline{m(p)}$.

With Lemma A.1 in mind it is easy to show that, for the DtN map in the simple case of zero boundary perturbation, i.e.

$$
T_{0}[\psi]=-k b \frac{d_{z} H_{D}^{(1)}(k b)}{H_{D}^{(1)}(k b)}[\psi],
$$

c.f. (10), the adjoint is

$$
T_{0}^{*}=\overline{-k b \frac{d_{z} H_{D}^{(1)}(k b)}{H_{D}^{(1)}(k b)}}=-k b \frac{d_{z} H_{D}^{(2)}(k b)}{H_{D}^{(2)}(k b)}=: S_{0} .
$$

Of course, $S_{0}^{*}=T_{0}$ as well, a fact that we will use later.

The goal of this section is to find a rapid OE formula for the evaluation of the DtN map. In the case of solving Laplace's equation $(k=0$ in (2)) one can take advantage of the fact that the DtN map is self-adjoint to take the adjoint of (19) and reverse the order of the operators. At first this would not appear to accelerate the procedure, but a careful inspection of (19) reveals that if the operator $T_{l}$ were applied to the same function $\psi$ at every order (rather than different ones) then these could be saved from one order to the next, resulting in an algorithm with the same computational complexity as the FE algorithm.

Of course $S_{0} \neq T_{0}$ for $k \neq 0$ so this idea will not work directly for the DtN map associated to the Helmholtz equation. However, a modification of this idea will produce a fast algorithm. If $S$ is the adjoint of $T$ then, of course, $T$ is the adjoint of $S$. If we can find an algorithm analogous to (19) for $S$, then we could take the adjoint of $S_{n}$ (reversing the order of the operators) to find a fast algorithm for $T_{n}$. With this in mind we now have three tasks: Find the problem for $S$, find the OE method for $S$, and conjugate this OE method.

For the first let us consider (7) and (8) and identify the adjoint of $T$. To begin, define another transparent boundary at $r=c$ which is exterior to $\mathcal{B}$. Following the development of Sect. 2.1 it is easy to see that (7) can be equivalently stated as

$$
\begin{aligned}
\Delta w+k^{2} w=0 & \text { in } \mathcal{A}_{\mathcal{B}, c}:=\operatorname{Ext}(\mathcal{B}) \cap\{r<c\} \\
w=\psi & \text { at } \mathcal{B} \\
\partial_{r} w-J w=0 & \text { at } r=c,
\end{aligned}
$$

where

$$
J:=c k \frac{d_{z} H_{D}^{(1)}(k c)}{H_{D}^{(1)}(k c)} .
$$


Note the opposite sign of $J$ as compared to $T$ since the outward direction is now the positive radial direction. We propose the "adjoint problem"

$$
\begin{aligned}
\Delta u+k^{2} u=0 & \text { in } \mathcal{A}_{\mathcal{B}, c} \\
u=\eta & \text { at } \mathcal{B} \\
\partial_{r} u-K u=0 & \text { at } r=c,
\end{aligned}
$$

where $K$ is to be determined, which defines the "adjoint" of the DtN map, $T$,

$$
S[\eta]:=\left.\nabla u\right|_{\mathcal{B}} \cdot N .
$$

We will now make the notion of (21)-(22) as adjoint of (7)-(8) more precise. We begin with the calculation

$$
\langle T[\psi], \eta\rangle=\int_{0}^{2 \pi} T[\psi] \bar{\eta} d \theta=\int_{0}^{2 \pi}\left(\partial_{n} w\right)|N| \bar{u} d \theta=\int_{\mathcal{B}}\left(\partial_{n} w\right) \bar{u} d s,
$$

where $n$ is the unit normal. It is here that we see how the particular choice of normal, $N$, is crucial to our adjointness arguments. Continuing via the divergence theorem (applied to the bounded domain $\mathcal{A}_{\mathcal{B}, c}$ ):

$$
\begin{aligned}
\langle T[\psi], \eta\rangle= & \int_{\mathcal{A}_{\mathcal{B}, c}} \operatorname{div}[\nabla w \bar{u}] d V-\int_{r=c}\left(\partial_{r} w\right) \bar{u} d s \\
= & \int_{\mathcal{A}_{\mathcal{B}, c}} \Delta w \bar{u} d V+\int_{\mathcal{A}_{\mathcal{B}, c}} \nabla w \cdot \overline{\nabla u} d V-\int_{r=c}\left(\partial_{r} w\right) \bar{u} d s \\
= & \int_{\mathcal{A}_{\mathcal{B}, c}}-k^{2} w \bar{u} d V+\int_{\mathcal{A}_{\mathcal{B}, c}} \operatorname{div}[w \overline{\nabla u}] d V \\
& -\int_{\mathcal{A}_{\mathcal{B}, c}} w \overline{\Delta u} d V-\int_{r=c}\left(\partial_{r} w\right) \bar{u} d s,
\end{aligned}
$$

where we have used the fact that $w$ satisfies the Helmholtz equation. Using the reality of $k$, the fact that $u$ satisfies the Helmholtz equation, and, again, the divergence theorem:

$$
\begin{aligned}
\langle T[\psi], \eta\rangle= & \int_{\mathcal{A}_{\mathcal{B}, c}}-k^{2} w \bar{u} d V+\int_{\mathcal{B}} w \overline{\partial_{n} u} d s+\int_{r=c} w \overline{\partial_{r} u} d V \\
& -\int_{\mathcal{A}_{\mathcal{B}, c}} w\left(-k^{2}\right) \bar{u} d V-\int_{r=c}\left(\partial_{r} w\right) \bar{u} d s .
\end{aligned}
$$

The first and fourth term cancel leaving:

$$
\langle T[\psi], \eta\rangle=\langle\psi, S[\eta]\rangle+\left\langle\left. w\right|_{r=c}, K\left[\left.u\right|_{r=c}\right]\right\rangle-\left\langle J\left[\left.w\right|_{r=c}\right],\left.u\right|_{r=c}\right\rangle .
$$

Clearly, $S$ is the adjoint of $T$ provided that $K$ is the adjoint of $J$ which implies

$$
K:=c k \frac{d_{z} H_{D}^{(2)}(k c)}{H_{D}^{(2)}(k c)},
$$

from Lemma A.1. 
Following the development of Sect. 3.2 it is easy to find OE recursions for the adjoint operator $S$. We begin with

$$
u_{p}(r, \theta)=H_{p}^{(2)}(k r) e^{i p \theta},
$$

which satisfies the Helmholtz equation and the condition at $r=c$, and insert this into the definition of $S$ yielding

$$
\begin{aligned}
S(\varepsilon f)\left[H_{p}^{(2)}(k(b+\varepsilon f)) e^{i p \theta}\right]= & -\left.(b+\varepsilon f) \partial_{r}\left(H_{p}^{(2)}(k r) e^{i p \theta}\right)\right|_{r=b+\varepsilon f} \\
& +\left.\frac{\varepsilon \partial_{\theta} f}{b+\varepsilon f} \partial_{\theta}\left(H_{p}^{(2)}(k r) e^{i p \theta}\right)\right|_{r=b+\varepsilon f}
\end{aligned}
$$

For the expansion $S(\varepsilon f)=\sum_{n=0}^{\infty} S_{n}(f) \varepsilon^{n}$, we have

$$
S_{0}[\eta]=-b k \frac{d_{z} H_{D}^{(2)}(k b)}{H_{D}^{(2)}(k b)}[\eta],
$$

and, using $\partial_{\theta}^{*}=-\partial_{\theta}$,

$$
\begin{aligned}
S_{n}(f)[\eta]= & -b k C_{n} \frac{d_{z}^{n+1} H_{D}^{(2)}(k b)}{H_{D}^{(2)}(k b)}[\eta]-k f C_{n-1} \frac{d_{z}^{n} H_{D}^{(2)}(k b)}{H_{D}^{(2)}(k b)}[\eta] \\
& +\sum_{l=0}^{n-1} C_{n-1-l} \frac{\left(\partial_{\theta} f\right)(-1)^{l} f^{l}}{b^{l+1}} \partial_{\theta} \frac{d_{z}^{n-1-l} H_{D}^{(2)}(k b)}{H_{D}^{(2)}(k b)}[\eta] \\
& -\sum_{l=0}^{n-1} S_{l}(f)\left[C_{n-l} \frac{d_{z}^{n-l} H_{D}^{(2)}(k b)}{H_{D}^{(2)}(k b)}[\eta]\right] .
\end{aligned}
$$

Defining

$$
M_{n}(D):=\frac{d_{z}^{n} H_{D}^{(2)}(k b)}{H_{D}^{(2)}(k b)},
$$

and taking the adjoint, we find

$$
S_{0}^{*}[\eta]=-b k \frac{d_{z} H_{D}^{(1)}(k b)}{H_{D}^{(1)}(k b)}[\eta]=T_{0}[\eta],
$$

and

$$
\begin{aligned}
S_{n}^{*}(f)[\eta]= & -b k M_{n+1}^{*}\left[C_{n} \eta\right]-k M_{n}^{*}\left[f C_{n-1} \eta\right] \\
& -\sum_{l=0}^{n-1} M_{n-1-l}^{*} \partial_{\theta}\left[C_{n-1-l} \frac{\left(\partial_{\theta} f\right)(-1)^{l} f^{l}}{b^{l+1}} \eta\right] \\
& -\sum_{l=0}^{n-1} M_{n-l}^{*}\left[C_{n-l} S_{l}^{*}(f)[\eta]\right] .
\end{aligned}
$$


Since $S_{n}^{*}=T_{n}$ and

$$
M_{n}^{*}=\frac{d_{z}^{n} H_{D}^{(1)}(k b)}{H_{D}^{(1)}(k b)},
$$

this provides a rapid formula for the computation of the $T_{n}$ :

$$
\begin{aligned}
T_{n}(f)[\psi]= & -b k \frac{d_{z}^{n+1} H_{D}^{(1)}(k b)}{H_{D}^{(1)}(k b)}\left[C_{n} \psi\right]-k \frac{d_{z}^{n} H_{D}^{(1)}(k b)}{H_{D}^{(1)}(k b)}\left[f C_{n-1} \psi\right] \\
& -\sum_{l=0}^{n-1} \frac{d_{z}^{n-1-l} H_{D}^{(1)}(k b)}{H_{D}^{(1)}(k b)} \partial_{\theta}\left[C_{n-1-l} \frac{\left(\partial_{\theta} f\right)(-1)^{l} f^{l}}{b^{l+1}} \psi\right] \\
& -\sum_{l=0}^{n-1} \frac{d_{z}^{n-l} H_{D}^{(1)}(k b)}{H_{D}^{(1)}(k b)}\left[C_{n-l} T_{l}(f)[\psi]\right]
\end{aligned}
$$

as the operators $T_{l}$ are now applied solely to the function $\psi$ and may be stored at every order.

\section{References}

1. Baker, G.A., Jr., Graves-Morris, P.: Padé Approximants, 2nd edn. Cambridge University Press, Cambridge (1996)

2. Bruno, O.P., Reitich, F.: Numerical solution of diffraction problems: A method of variation of boundaries. J. Opt. Soc. Am. A 10(6), 1168-1175 (1993)

3. Bruno, O.P., Reitich, F.: Numerical solution of diffraction problems: A method of variation of boundaries. II. Finitely conducting gratings, Padé approximants, and singularities. J. Opt. Soc. Am. A 10(11), 23072316 (1993)

4. Bruno, O.P., Reitich, F.: Numerical solution of diffraction problems: A method of variation of boundaries. III. Doubly periodic gratings. J. Opt. Soc. Am. A 10(12), 2551-2562 (1993)

5. Bruno, O.P., Reitich, F.: Calculation of electromagnetic scattering via boundary variations and analytic continuation. Appl. Comput. Electromagn. Soc. J. 11(1), 17-31 (1996)

6. Bruno, O.P., Reitich, F.: Boundary-variation solutions for bounded-obstacle scattering problems in three dimensions. J. Acoust. Soc. Am. 104(5), 2579-2583 (1998)

7. Bruno, O.P., Reitich, F.: High-order boundary perturbation methods. In: Mathematical Modeling in Optical Science. Frontiers in Applied Mathematics Series, vol. 22, pp. 71-109. SIAM, Philadelphia (2001)

8. Colton, D., Kress, R.: Inverse Acoustic and Electromagnetic Scattering Theory, 2nd edn. Springer, Berlin (1998)

9. Demkowicz, L., Ihlenburg, F.: Analysis of a coupled finite-infinite element method for exterior Helmholtz problems. Numer. Math. 88(1), 43-73 (2001)

10. Feng, K.: Finite element method and natural boundary reduction. In: Proceedings of the International Congress of Mathematicians (Warsaw, 1983), vols. 1, 2, pp. 1439-1453. PWN, Warsaw (1984)

11. Givoli, D.: Nonreflecting boundary conditions. J. Comput. Phys. 94(1), 1-29 (1991)

12. Givoli, D.: Numerical Methods for Problems in Infinite Domains. Studies in Applied Mechanics, vol. 33. Elsevier, Amsterdam (1992)

13. Givoli, D.: Recent advances in the DtN FE method. Arch. Comput. Methods Eng. 6(2), 71-116 (1999)

14. Givoli, D., Keller, J.B.: Special finite elements for use with high-order boundary conditions. Comput. Methods Appl. Mech. Eng. 119(3-4), 199-213 (1994)

15. Grote, M.J., Keller, J.B.: On nonreflecting boundary conditions. J. Comput. Phys. 122(2), 231-243 (1995)

16. Han, H.D., Wu, X.N.: Approximation of infinite boundary condition and its application to finite element methods. J. Comput. Math. 3(2), 179-192 (1985)

17. Harari, I., Hughes, T.J.R.: Analysis of continuous formulations underlying the computation of timeharmonic acoustics in exterior domains. Comput. Methods Appl. Mech. Eng. 97(1), 103-124 (1992) 
18. Hu, B., Nicholls, D.P.: Analyticity of Dirichlet-Neumann operators on Hölder and Lipschitz domains. SIAM J. Math. Anal. 37(1), 302-320 (2005)

19. Ihlenburg, F.: Finite Element Analysis of Acoustic Scattering. Springer, New York (1998)

20. Keller, J.B., Givoli, D.: Exact nonreflecting boundary conditions. J. Comput. Phys. 82(1), 172-192 (1989)

21. Lord Rayleigh: On the dynamical theory of gratings. Proc. R. Soc. Lond. A 79, 399-416 (1907)

22. Milder, D.M.: An improved formalism for rough-surface scattering of acoustic and electromagnetic waves. In: Proceedings of SPIE-The International Society for Optical Engineering (San Diego, 1991), vol. 1558, pp. 213-221. SPIE, Bellingham (1991)

23. Milder, D.M.: An improved formalism for wave scattering from rough surfaces. J. Acoust. Soc. Am. 89(2), 529-541 (1991)

24. Milder, D.M.: An improved formalism for electromagnetic scattering from a perfectly conducting rough surface. Radio Sci. 31(6), 1369-1376 (1996)

25. Milder, D.M.: Role of the admittance operator in rough-surface scattering. J. Acoust. Soc. Am. 100(2), 759-768 (1996)

26. Milder, D.M., Sharp, H.T.: Efficient computation of rough surface scattering. In: Mathematical and Numerical Aspects of Wave Propagation Phenomena (Strasbourg, 1991), pp. 314-322. SIAM, Philadelphia (1991)

27. Milder, D.M., Sharp, H.T.: An improved formalism for rough surface scattering. II: Numerical trials in three dimensions. J. Acoust. Soc. Am. 91(5), 2620-2626 (1992)

28. Nicholls, D.P., Nigam, N.: Exact non-reflecting boundary conditions on general domains. J. Comput. Phys. 194(1), 278-303 (2004)

29. Nicholls, D.P., Nigam, N.: Error analysis of a coupled finite element/DtN map algorithm on general domains. Numer. Math. 105(2), 267-298 (2006)

30. Nicholls, D.P., Reitich, F.: Stability of high-order perturbative methods for the computation of DirichletNeumann operators. J. Comput. Phys. 170(1), 276-298 (2001)

31. Nicholls, D.P., Reitich, F.: Shape deformations in rough surface scattering: Cancellations, conditioning, and convergence. J. Opt. Soc. Am. A 21(4), 590-605 (2004)

32. Nicholls, D.P., Reitich, F.: Shape deformations in rough surface scattering: Improved algorithms. J. Opt. Soc. Am. A 21(4), 606-621 (2004)

33. Rice, S.O.: Reflection of electromagnetic waves from slightly rough surfaces. Commun. Pure Appl. Math. 4, 351-378 (1951)

34. Warburton, T., Pavarino, L.F., Hesthaven, J.S.: A pseudo-spectral scheme for the incompressible NavierStokes equations using unstructured nodal elements. J. Comput. Phys. 164(1), 1-21 (2000) 